\title{
ArcheoSciences
}

Revue d'archéométrie

\section{Nouvelles données sur la position du littoral antique de Fréjus. Le diagnostic archéologique du « théâtre d'agglomération » (Fréjus, Var)}

New data on the ancient littoral of Fréjus. The archaeological evaluation of the «théâtre d'agglomération » (Fréjus, Var)

Pierre Excoffon, Benoît Devillers, Stéphane Bonnet et Laurent Bouby

\section{OpenEdition \\ Journals}

Édition électronique

URL : https://journals.openedition.org/archeosciences/382

DOI : 10.4000/archeosciences.382

ISBN : 978-2-7535-1595-6

ISSN : 2104-3728

Éditeur

Presses universitaires de Rennes

\section{Édition imprimée}

Date de publication : 31 décembre 2006

Pagination : 205-221

ISBN : 978-2-7535-0456-1

ISSN : 1960-1360

Référence électronique

Pierre Excoffon, Benoît Devillers, Stéphane Bonnet et Laurent Bouby, « Nouvelles données sur la position du littoral antique de Fréjus. Le diagnostic archéologique du «théâtre d'agglomération » (Fréjus, Var) », ArcheoSciences [En ligne], 30 | 2006, document 20, mis en ligne le 31 décembre 2008, consulté le 01 février 2022. URL : http://journals.openedition.org/archeosciences/382 ; DOI : https:// doi.org/10.4000/archeosciences.382 


\title{
Nouvelles données sur la position du littoral antique de Fréjus. Le diagnostic archéologique du " théâtre d'agglomération » (Fréjus, Var)
}

\author{
Pierre Excoffon* et Benoît Devillers** \\ avec la collaboration de Stéphane Bonnet*** et Laurent Bouby**
}

\begin{abstract}
Résumé : En juillet 2005, s'est déroulée une campagne de sondages archéologiques sur le site du futur théâtre d'agglomération de Fréjus/SaintRaphaël sur la commune de Fréjus. Pour ce projet, une équipe pluridisciplinaire a été constituée, associant archéologues et géomorphologues. Les tranchées réalisées ont permis de mettre au jour une portion de côte rocheuse, qui a été dégagée sur environ $40 \mathrm{~m}^{2}$. Ainsi, cette découverte a permis de faire reculer le positionnement du trait de côte de près d'un kilomètre par rapport aux données récentes publiées. Vers le milieu du $\mathrm{I}^{\text {er }}$ siècle av. J.-C., au moment de la création de Forum Iulii, le littoral se présentait au sud de la Butte Saint-Antoine, sous la forme d'une bande étroite d'environ $100 \mathrm{~m}$ de large, ponctuée au sud par une pointe rocheuse. Lors de cette opération, ont également été mises en évidence l'importance et la rapidité du phénomène de progradation, transformant en près d'un siècle une côte rocheuse étroite en une vaste arrière plage. En effet, le $\mathrm{I}^{\text {er }}$ siècle marque l'avancée rapide du cordon littoral sur le site sous l'effet des apports sédimentaires de l'Argens. Les terrains gagnés sur la mer sont occupés, mais aucune trace de constructions n’a été repérée.
\end{abstract}

\begin{abstract}
In July 2005, was held an archaeological survey campaign on the site of the future theatre of agglomeration of Fréjus/Saint-Raphaël on the commune of Frejus. For this project, a multi-field team was set up, associating archaeologists and sedimentologists. The trenches carried out revealed a portion of rock shore coast, which was released on approximately 40 square metres. Thus, this discovery allowed us to move back the existing coastline of almost one kilometre into the land compared to the recent data published. In the middle of Ist century A.D., at the time of the creation of Forum Iulii, this littoral was shaped by a narrow band of approximately $100 \mathrm{~m}$ wide at the south of the Butte Saint-Antoine, delimited by a rock point in the south. During this operation, the importance and the speed of the phenomenon of progradation were also highligthed, transforming into nearly one century a narrow rock coast into a vast back beach.
\end{abstract}

Mots clés : Cordon littoral, côte rocheuse, Fréjus, niveau marin, période romaine, port, progradation, géoarchéologie.

Key words : Fréjus, marine level, offshore bar, port, progradation, Roman period, rocky coast, Geoarchaeology.

\footnotetext{
* Service du Patrimoine de la Ville de Fréjus, 51 rue Aubenas, 83600 Fréjus. (p.excoffon@voila.fr)

** CEPAM, UMR CNRS 6130, 250 rue Albert-Einstein, Sophia Antipolis, 06560 Valbonne.(devillers@cepam.cnrs.fr)

*** Centre Européen de Recherche et d'Enseignement des Géosciences de l'Environnement, UMR CNRS 6635, Europôle méditerranéen de l'Arbois, BP 80, 13545 Aix-en-Provence cedex 04.
} 


\section{Présentation}

\subsection{Localisation dans la ville antique}

Durant le mois de juillet 2005, le Service du Patrimoine de la Ville de Fréjus a réalisé un diagnostic archéologique sur le site du futur théâtre d'agglomération, sur la commune de Fréjus. Dans le cadre de cette opération, financée par la Communauté d'Agglomération de Fréjus/Saint-Raphaël, une équipe pluridisciplinaire a été constituée, associant des archéologues et des géomorphologues ${ }^{1}$.

Le terrain sondé se situe à $81 \mathrm{~m}$ au sud du promontoire rocheux de la Butte Saint-Antoine (Fig. 1 [1]), à environ $150 \mathrm{~m}$ du quai méridional du port antique et à $1,3 \mathrm{~km}$ de la côte actuelle.

Le port antique [2], distant de la côte actuelle de $1500 \mathrm{~m}$, occupe une superficie de près de 17 ha. Le quai méridional est le mieux conservé : depuis son extrémité occidentale à l'est de la Butte Saint-Antoine, on le suit le long du Chemin de la Lanterne sur environ $560 \mathrm{~m}$, jusqu'à la Lanterne d'Auguste. Ce monument se trouve au point de contact entre le quai méridional et le chenal d'accès au port à l'est. Il est le plus souvent interprété comme un amer signalant l'entrée du port. Le canal d'accès est matérialisé par un mur de protection (crénelé?) long de $220 \mathrm{~m}$, marquant le quai occidental du canal. Le pendant de ce quai, comme le prolongement du canal vers la mer n'est restituable qu'à partir de photographies aériennes et par l'étude du cadastre (février 1963).

Sur le Chemin de la Lanterne d'Auguste [3], au pied de la Butte Saint-Antoine, Charles Texier signale déjà au XIX ${ }^{\mathrm{e}}$ siècle l'existence de vestiges extra-muros, fait confirmé en 1994 par la découverte de murs et de mosaïques attestant la présence d'un habitat daté entre le début du $\mathrm{I}^{\text {er }}$ et le III $^{\mathrm{e}}$ siècle (Moerman 1994). Ces vestiges, localisés dans une tranchée étroite, sont à moins de $80 \mathrm{~m}$ au nord de la zone concernée par les diagnostics. Quelques dizaines de mètre plus à l'ouest [4], un mur en petit appareil de $45 \mathrm{~m}$ de long a été découvert par I. Béraud en 1991 le long du boulevard Severin Decuers. L'extrémité sud de ce mur n'est distante des sondages que de $70 \mathrm{~m}$.

$\mathrm{Au}$ niveau du rond-point de la Miougrano [5], Cl. Varoqueaux a mis au jour quelques tombes non précisément datées (Antiquité tardive?). Celles-ci se trouvent seulement à une vingtaine de mètres du diagnostic. Entre la zone étudiée

1. L'équipe, sous la direction de Pierre Excoffon, était constituée par Sandrine Ardisson (UNSA/CEPAM), Stéphane Bonnet (CEREGE), Benoît Devillers (CEPAM), Sandra Greck (IMEP), Romuald Mercurin et Joris Pâques (Service Archéologique, Ville de Fréjus). Sur le chantier, nous avons également bénéficié des conseils éclairés de Michel Pasqualini, Chérine Gébara et Christophe Morhange. et le rivage actuel, aucun vestige de la période antique n’a à ce jour été identifié.

Enfin, à partir d'une photographie aérienne de 1944 (Fig. 2), G. Chouquer et Ch. Gébara ont proposé l'existence d'un canal artificiel romain assurant une relation entre le port de Fréjus et l'embouchure de l'Argens (Gébara et Chouquer 1996). Cette hypothèse s'appuyée sur la présence d'une bande sombre large de 14 à $20 \mathrm{~m}$ passant au sud du quartier de Villeneuve et se prolongeant en direction du bassin du port romain. Le tracé, qui se situe en partie dans la zone étudiée, fût interprété comme un second chenal d'accès au port. Cette hypothèse, nous le verrons, doit être définitivement écartée.

\subsection{Prémices et fondations de la colonie romaine}

Bien que [très] discutée, la date de fondation de Forum Iulii est antérieure à 43 av. J.-C., mais probablement pas à 49 (Gascou \& Janon 1985, 14-15). On ne connaît pour ainsi dire rien de la forme de ce premier Forum, étape sur la route d'Italie vers l'Espagne, ni de sa position exacte. Après la bataille d'Actium en septembre 31 av. J.-C., les navires d'Antoine et leurs équipages capturés par Octave sont envoyés à Fréjus. C'est peut-être à ce moment qu'est édifié ce qui est interprété comme un camp militaire à Villeneuve (Goudineau 1982). Cette zone se trouve à moins de $600 \mathrm{~m}$ à l'ouest du site du théâtre d'agglomération et devait ellemême se trouver à proximité de la mer. Fréjus devient quelques années plus tard, entre 29 et 27 av. J.-C., une colonie de droit romain avec la déduction de vétérans de la VIII légion. C'est alors que la localité commence réellement à se développer, avec des campagnes organisées et un début d'urbanisme que l'on perçoit encore assez mal, suivant le premier réseau urbain. C'est à partir de cette époque que l'on doit situer les plus anciennes traces de fréquentation repérées dans ce sondage, donc peu après la déduction de la colonie, période où se produit une forte augmentation de la population.

\subsection{Géologie}

Le site se trouve à la retombée de l'extrémité méridionale, en l'occurrence la Butte Saint-Antoine, d'un ensemble géologique appelée formation du Mitan ${ }^{2}$. Celle-ci se caractérise au niveau de Fréjus par des grès bruns ou roses permiens, parfois des arkoses conglomératiques, alternant avec des pélites. La Butte culmine à $11 \mathrm{~m} \mathrm{NGF}$ et sur les fouilles du

2. Carte géologique de la France à 1/50000', Fréjus-Cannes, Edition du BRGM, 1994, 51-52. 


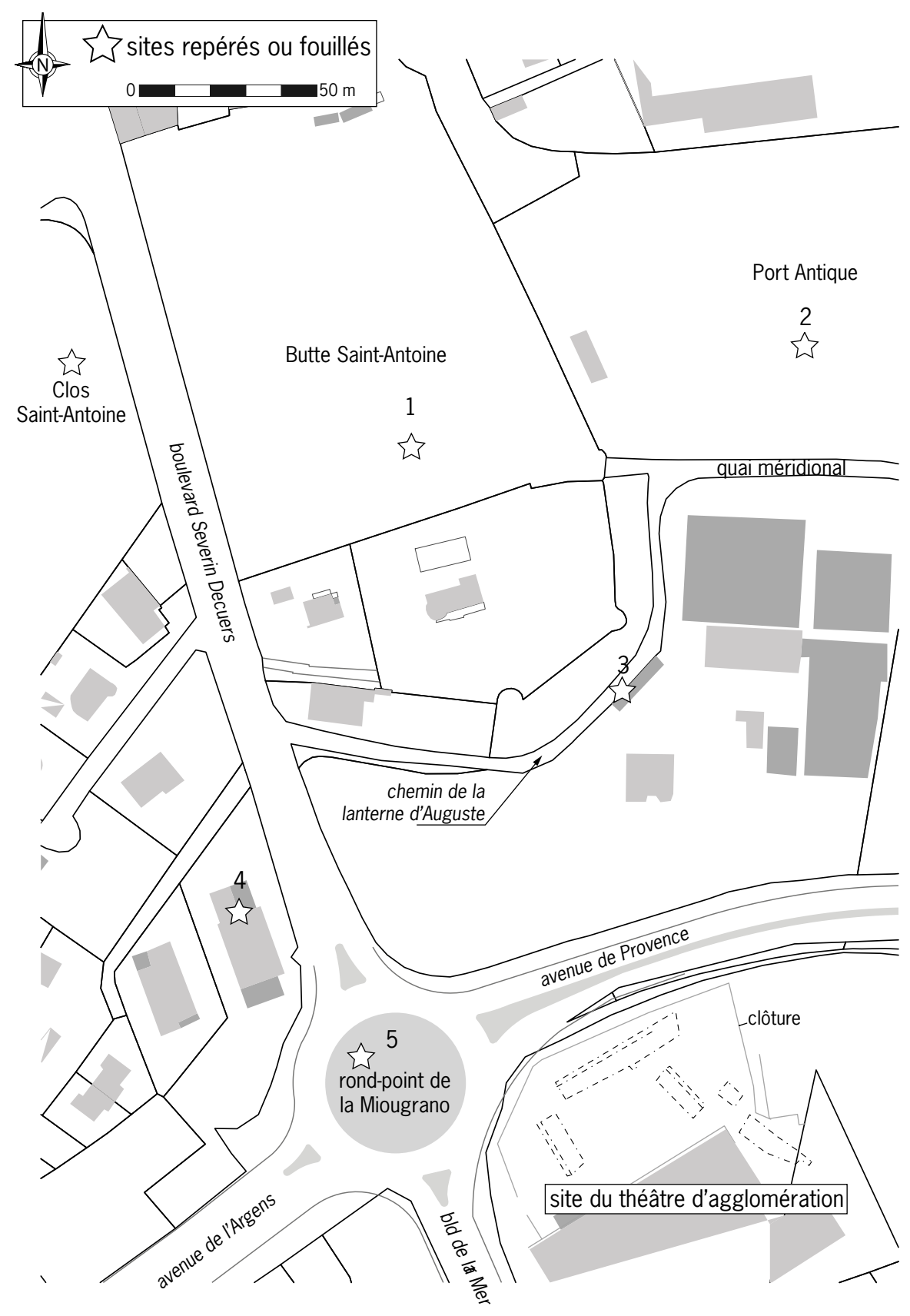

Figure 1 : Localisation de l'opération du théâtre d'agglomération et contexte archéologique environnant (J. Pâques/F. laurier).

Figure 1: Localization of the "théatre d'agglomération" operation and surrounding archaeological context.

théâtre agglomération, le point le plus bas du rocher a été atteint à une profondeur de -1,20 m NGF. Localement cette roche permienne constitue le réceptacle des sédiments meubles holocènes qui ont comblé la vallée de l'Argens depuis plus de 6000 ans par progradation deltaïque pour donner lieu à ce paysage de ria (Dubar 2004).

\subsection{Organisation du chantier}

Le terrain concerné couvre une superficie de $3300 \mathrm{~m}^{2}$. Il est limité au nord par l'Avenue de Provence, au sud par la rue du Mas, à l'est par les constructions abritant les services administratifs de la ville et à l'ouest par le boulevard de la

ArcheoSciences, revue d'archéométrie, 30, 2006, p. 205-221 


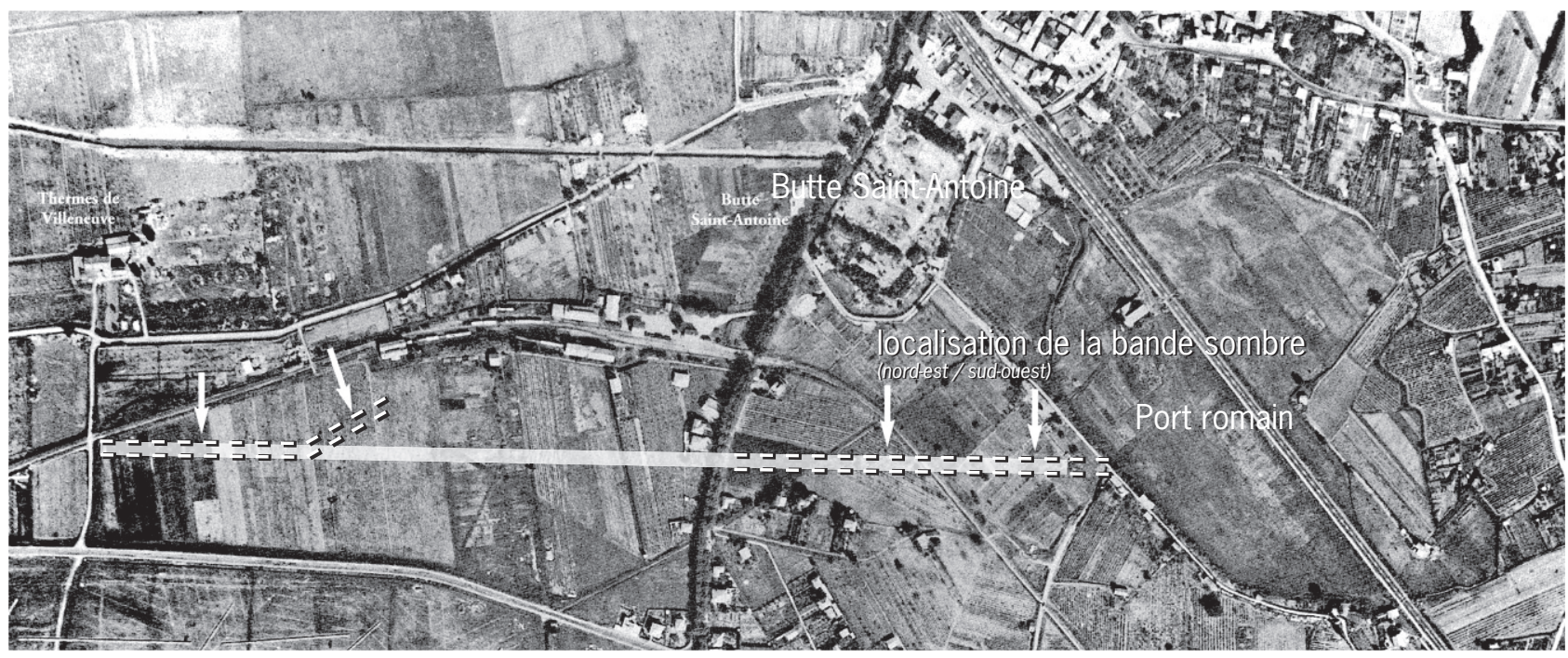

Figure 2 : Photo aérienne du secteur situé entre le quartier Villeneuve et le port antique. En surimpression reconstitution du tracé du canal hypothétique, les flèches indiquent la localisation de la trace sombre (Mission américaine du 03 août 1944; cliché 682 DR.145, $\mathrm{n}^{\circ}$ 3009, fonds Ecole Française de Rome, en dépôt au Centre Camille-Jullian).

Figure 2: Air photograph of the sector located between the Villeneuve district and the ancient port. As overprinting it is the reconstitution of the layout of the hypothetical channel, the arrows indicate the localization of the dark trace (American Mission of August 03, 1944, photograph 682 DR.145, 3009).

Mer (Fig. 1). Cinq tranchées ont été réalisées (Fig. 3). Afin de faire apparaitre en coupe des séquences stratigraphiques significatives, il était primordial de descendre suffisamment profond et sur une longueur importante. La nature des sables, la puissance de ces dépôts et leur disposition n'avaient jamais pu être observées en coupe profonde. La nature des sols, ainsi que la position de la nappe phréatique (entre deux et trois mètres selon les saisons) déterminées à partir des études géotechniques réalisées en amont du projet de construction (forages et sondages) ne nous permettaient pas de pratiquer des tranchées au-delà de $1,30 \mathrm{~m}$ de profondeur. Nous avons alors opté pour la technique des "well-point ", c'est-à-dire d'un rabattement de la nappe phréatique. Les parois des tranchées ont été étagées et talutées, tout en laissant par endroits des parements suffisamment verticaux pour la réalisation des coupes.

\subsection{Problématique et méthodologie}

La localisation des diagnostics à proximité du port antique dans une zone au contact de la roche permienne et des terrains atterris depuis l'antiquité, a été l'opportunité pour nous d'observer dans de profonds sondages et dans de larges coupes la situation du littoral dans l'antiquité. Plusieurs sondages et carottages réalisés aux alentours ${ }^{3}$ permettaient

3. Sondages INRAP au sud des thermes de Villeneuve en 2005 (J.M. Michel), à la Résidence Magalie et au Clos des Vignes en 2004 (A. d'émettre certains doutes sur la position réelle du bord de mer au début de l'existence de la colonie. Cette hypothèse se trouvait confortée par l'absence de vestige reconnu entre le secteur du futur théâtre d'agglomération et le rivage actuel.

L’approche adoptée, résolument géoarchéologique, s'est divisée en deux temps. Tout d'abord, l'étude des faciès anthropiques (dépôts à forte teneur en artefacts) et pédosédimentaires (lithofaciès, pédofaciès et biofaciès) a été réalisée sur le site. Il faut noter que la diversité des faciès rencontrés, leur grande lisibilité paléoenvironnementale et la grande extension des sondages ont permis une identification précise et fiable des paléomilieux sur le terrain. Ensuite, les enregistrements archéologiques et sédimentaires ont été complétés par une série d'analyses en laboratoire qui ont permis d'affiner et de valider les interprétations. Pour ce faire 45 analyses granulométriques ont été réalisées. Les biofaciès, autres indicateurs des paléomilieux, ont été identifiés au travers de la détermination de la macro-faune benthique (mollusques marins) ainsi que du contenu carpologique et xylologique des sédiments. En raison de l'importance des informations et de la redondance de certains résultats, l'ensemble des données ne sera pas exposé exhaustivement.

Dumont et $Y$. Meyer), ainsi que des carottages réalisés dans le port antique (F. Bertrand : Vitesse et processus de sédimentation intra et extra-lagunaire du port antique de Fréjus, Mémoire de Maîtrise, dir. Chr. Morhange, UFR de Géographie, Université Aix-Marseille 1, 2003, 68 p. et annexes) 


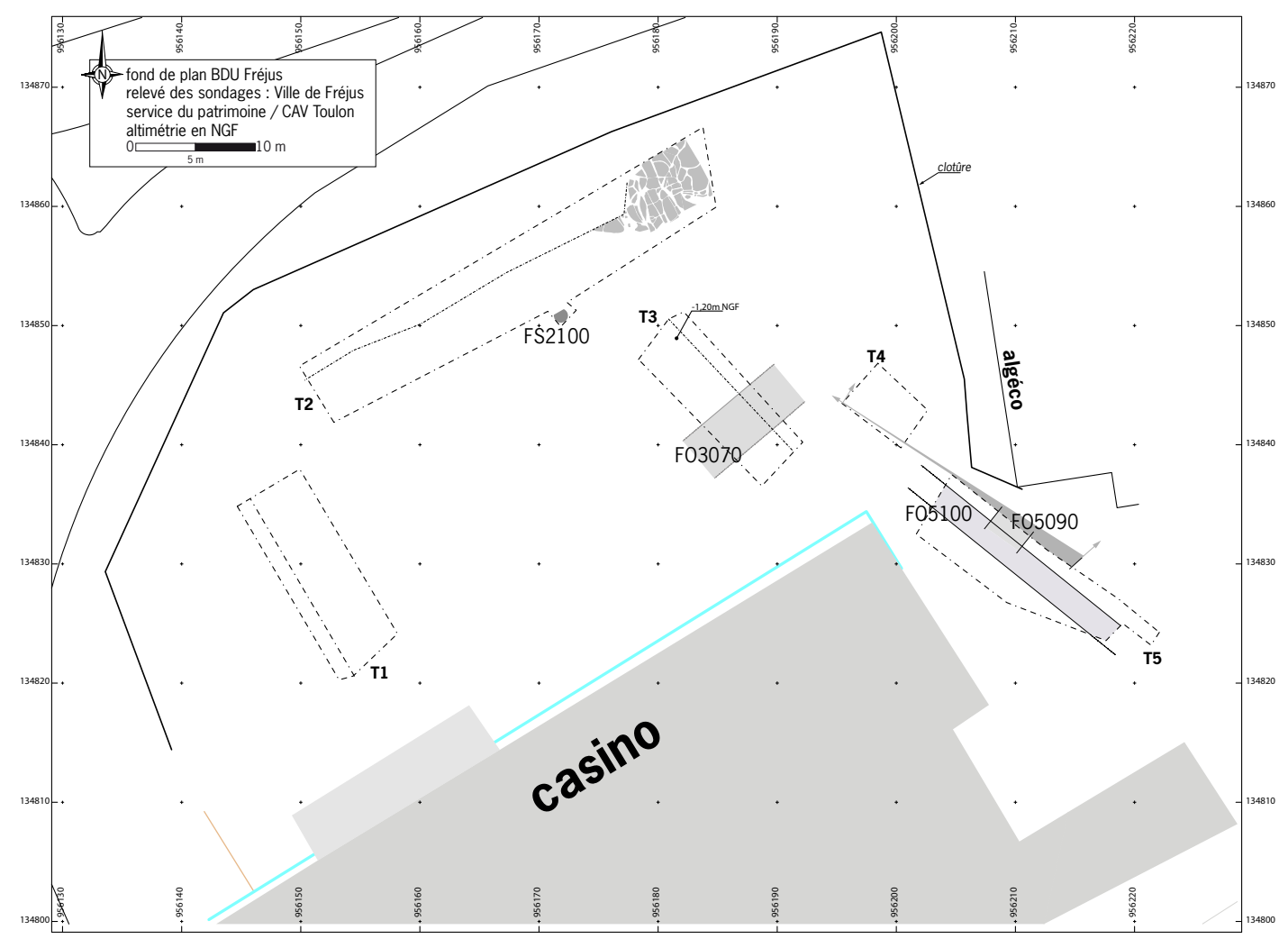

Figure 3 : Plan des cinq tranchées réalisées et principales découvertes (F. Laurier/J. Pâques).

Figure 3: Plan of the five trenches carried out and main discoveries.

Les niveaux rencontrés nous ont amenés à diviser l'étude de la fouille en cinq phases distinctes. Trois sont attribuables à la période antique, c'est celles-ci que nous présentons ici.

\section{DESCRIPTIONS ET INTERPRÉTATIONS ARCHÉOLOGIQUES ET PALÉOGÉOGRAPHIQUES}

\subsection{Phase $\mathrm{I}$ : le rivage au $\mathrm{I}^{\mathrm{er}}$ siècle avant $\mathrm{J}$.-C.}

\subsubsection{Description}

C'est à l'extrémité est de la tranchée 2 qu'un saillant de roche permienne a été découvert à partir de la côte $0,64 \mathrm{~m}$ NGF (Fig. 4 et 5). Sur une superficie d'environ $40 \mathrm{~m}^{2}$, nous avons découvert l'extrémité d'un enrochement de grès ocre-brun (US 2070) dont la surface est marquée par des cannelures profondes orientées nord-sud, remplies de galets, de faune marine et d'artefacts plus ou moins concrétionnés (US 2090). Ce saillant rocheux montre un étagement des formes d'érosion marine, on retrouve des formes d'abrasion marine (cannelures médiolittorales) dans sa partie haute et des cupules d'érosion infralittorales dans sa partie basse.
Outre quelques fragments de bois gorgés d'eau ${ }^{4}, 250$ fragments de céramiques, 14 de tegulae, sept d'imbrices, 21clous, une lame de couteau, un fragment d'enduit peint et cinq os ${ }^{5}$ ont été découverts. De nombreux fragments, notamment des panses d'amphores à huile de Bétique, ou encore un col de Dressel 7 /11 marseillaise, étaient littéralement coincés entre les failles du rocher, parfois très profondément (Fig. 6). Ce col montre par ailleurs des marques d'érosion partielle caractéristiques de contacts répétés avec une eau active, témoignant de son exposition au ressac (Fig. 7). Parmi les fragments découverts, nombreux étaient ceux portant des restes ou des traces de mollusques et de vers marins (Fig. 8). Ce matériel, piégé dans les infractuositées du rocher, témoigne d'un milieu médiolittoral, alternativement émergé et immergé sous l'effet des vagues et des micro-marées.

4. Seul un fragment de bois sans écorce, très érodé, de forme indéterminée et d'un volume approximatif de $100 \mathrm{~cm}^{3}$ a pu être déterminé. Il s'agit d'un Quercus ilex L., Chêne vert ou Quercus coccifera L., Chêne kermès. Nous devons cette identification à Sandra Greck (IMEP).

5. À cet ensemble s'ajoutent quelques ossements animaux : un bord de palette droite de porc, un fragment d'os zygomatique droit d'un crâne de porc, un talus droit de bœuf et une esquille indéterminée. Nous devons l'identification à Isabelle Rodet-Belarby (INRAP/CEPAM). 


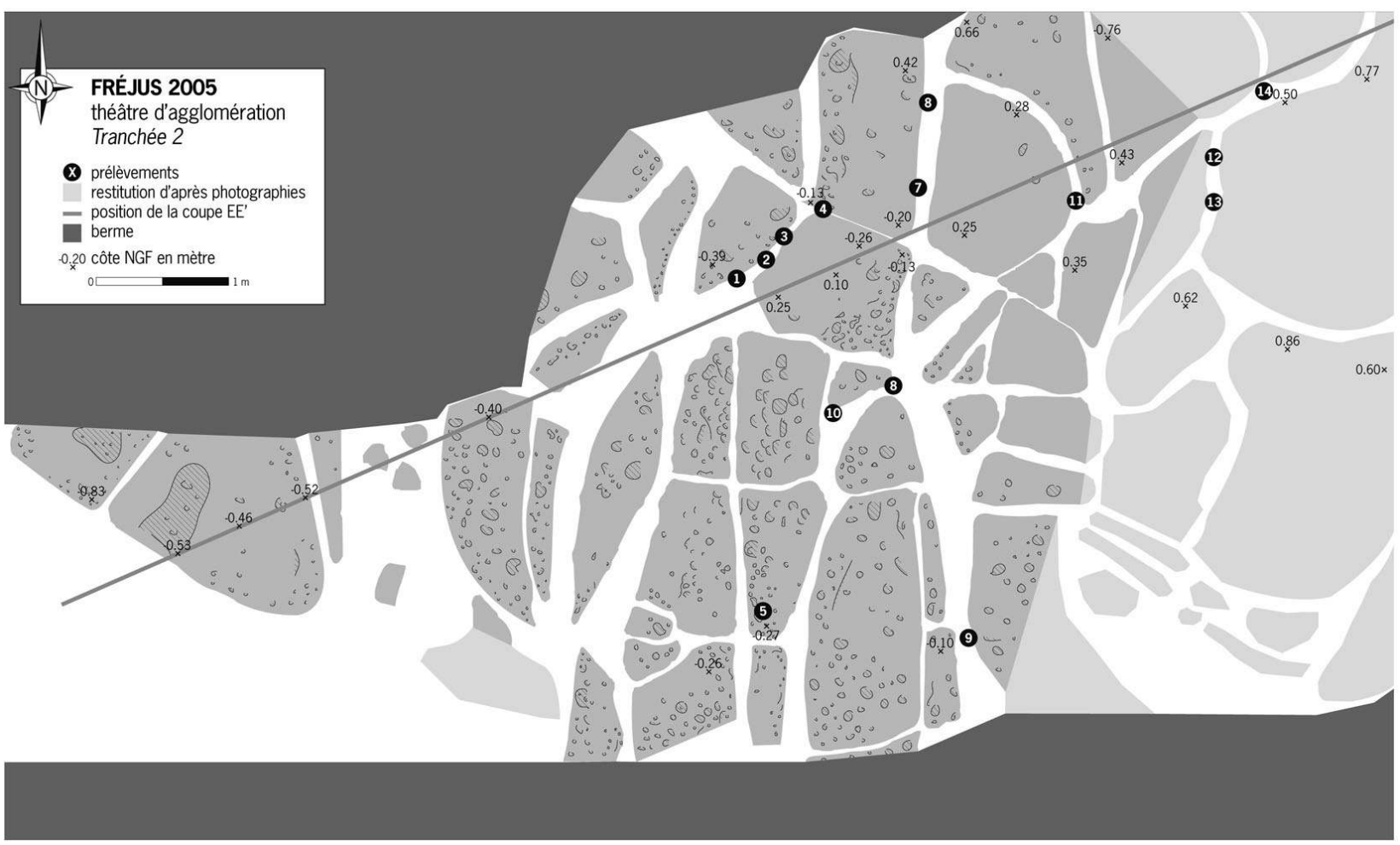

Figure 4 : Relevé d'une partie du promontoire rocheux (2070) (M. Pasqualini/P. Excoffon/J. Pâques). Figure 4: Record of a part of the rock headland.

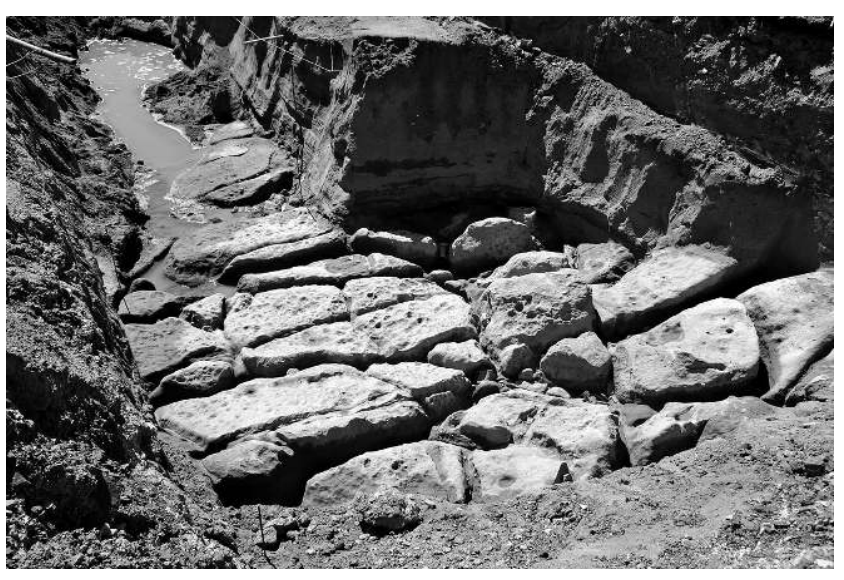

Figure 5 : Vue verticale oblique du rocher (P. Excoffon). Figure 5: Vertical sight of the rock.

La très forte proportion d'artefacts par rapport à la fraction lithoclastique et leur bon état de conservation (très partiellement émoussé) démontre qu’il ne s'agit pas de tessons amenés par la mer, mais bien de déchets délibérément jetés, notamment de très nombreux fragments de tuiles, assimilant cet ensemble à un véritable dépotoir et témoignant d'une importante pollution anthropique.

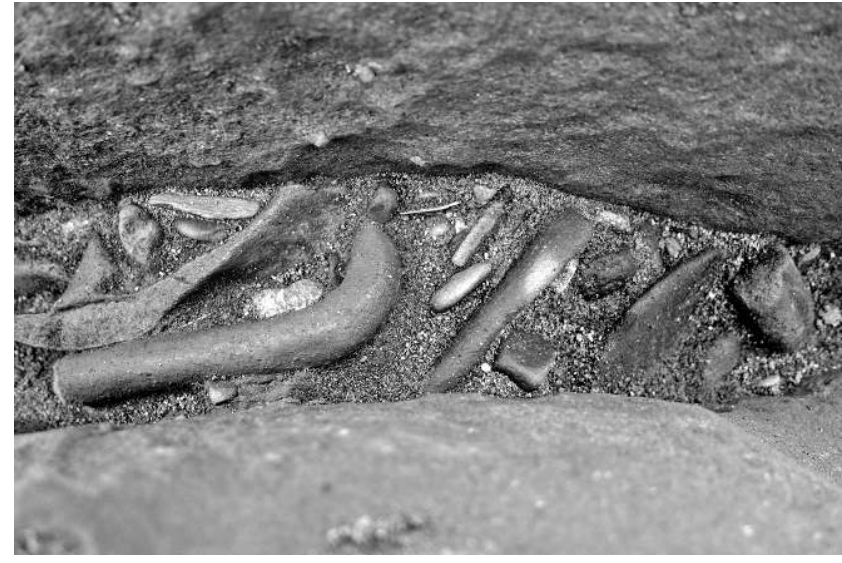

Figure 6 : Fragments d'amphores et galets pris entre les failles du rocher (P. Excoffon).

Figure 6: Amphoras fragments and rollers stuck in the faults of the rock.

\subsubsection{Le mobilier}

Le lot de mobilier découvert dans les failles du rocher (US2090) permet d'apporter une datation relativement précise pour la constitution de ce dépotoir (Fig. 9 et 10). 


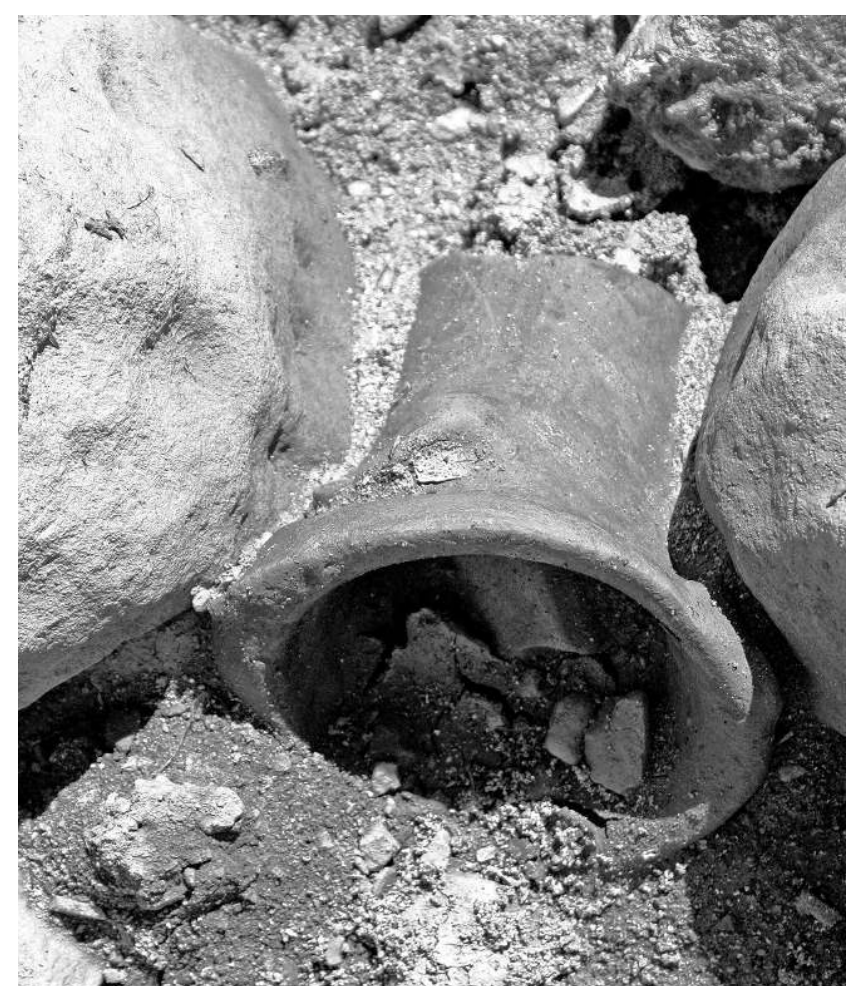

Figure 7 : Col d'amphore marseillaise (Dressel 7/11) partiellement érodé pris dans le rocher (P. Excoffon).

Figure 7: Marseilles amphora collar (Dressel 7/11) partially eroded stuck in the rock.

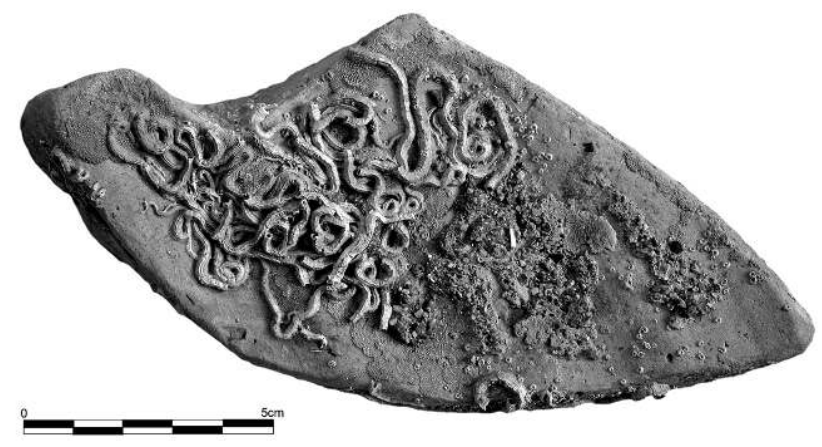

Figure 8 : Vers marins sur panse d'amphore (J. Pâques). Figure 8: Marin worms on amphora paunch.

La vaisselle

La vaisselle fine n'est représentée que par un seul fond de sigillée italique. Cette catégorie apparaît sur le littoral provençal à partir des années 30/20 av. J.-C. La vaisselle culinaire italique fournit un premier calage. La marmite 3c (Bats 1996) (Fig. 10 [1]), à bord horizontal, n'est pas diffusée au delà du changement d'ère, alors que le couvercle $7 \mathrm{c}$ serait en revanche plutôt caractéristique du i ${ }^{\text {er }}$ siècle apr. J.-C. (Fig. 10 [2] et [3]). L'apparition de la cruche ou bouilloire en pâte

\begin{tabular}{|c|c|c|c|c|}
\hline Zone 2 & US 2090 & & & \\
\hline Typologie & Catégorie & forme & NMI & Figures \\
\hline COM-IT 3c & \multirow{2}{*}{$\begin{array}{l}\text { céramique } \\
\text { commune } \\
\text { italique }\end{array}$} & $\begin{array}{c}\text { bord de } \\
\text { caccabus }\end{array}$ & 1 & 10. 1 \\
\hline COM-IT 7c & & $\begin{array}{l}\text { bords de } \\
\text { couvercle }\end{array}$ & 2 & 10. 2 et 3 \\
\hline KAOL F1 & $\begin{array}{c}\text { céramique } \\
\text { kaolinitique }\end{array}$ & bord de cruche & 1 & \\
\hline $\begin{array}{c}\text { CNT-VAR } \\
\text { Riv5a }\end{array}$ & $\begin{array}{c}\text { modelée } \\
\text { varoise }\end{array}$ & bord de plat & 1 & 10. 4 \\
\hline $\begin{array}{c}\text { CL-IMPRO } \\
\text { Pasq } 2\end{array}$ & \multirow{3}{*}{$\begin{array}{l}\text { Pâte claire } \\
\text { provençale }\end{array}$} & bord de coupe & 1 & 10. 5 \\
\hline $\begin{array}{c}\text { CL-IMPRO } \\
\text { Pasq A1 }\end{array}$ & & \multirow{2}{*}{$\begin{array}{l}\text { bords de } \\
\text { cruche }\end{array}$} & 1 & \\
\hline $\begin{array}{c}\text { CL-IMPRO } \\
\text { Pasq A1 }\end{array}$ & & & $\begin{array}{c}\text { bords de } \\
\text { cruche }\end{array}$ & \\
\hline SIG-IT & $\begin{array}{c}\text { céramique } \\
\text { sigillée italique }\end{array}$ & fond de vase & 1 & \\
\hline A-BET Dr20 & \multirow{3}{*}{$\begin{array}{l}\text { amphore de } \\
\text { Bétique }\end{array}$} & $\begin{array}{c}\text { tessons } \\
\text { d'amphores }\end{array}$ & 2 & \\
\hline \multirow[t]{2}{*}{ A-BET Dr7/11 } & & fond & 1 & \\
\hline & & $\begin{array}{c}\text { anses } \\
\text { d'amphores }\end{array}$ & 3 & \\
\hline A-TAR Dr2/4 & $\begin{array}{l}\text { amphore de } \\
\text { Tarraconaise }\end{array}$ & $\begin{array}{c}\text { anse } \\
\text { d'amphore }\end{array}$ & 1 & \\
\hline \multirow[t]{2}{*}{ A-GAUL Dr2/4 } & \multirow{2}{*}{$\begin{array}{l}\text { amphore } \\
\text { gauloise }\end{array}$} & $\begin{array}{c}\text { bord } \\
\text { d'amphore }\end{array}$ & 1 & 10. 6 \\
\hline & & $\begin{array}{c}\text { anses } \\
\text { d'amphores }\end{array}$ & 2 & \\
\hline A-ITI Dr2/4 & $\begin{array}{c}\text { amphore } \\
\text { italique } \\
\text { impériale }\end{array}$ & $\begin{array}{c}\text { bord } \\
\text { d'amphore }\end{array}$ & 1 & 10.7 \\
\hline A-M-I Dr7/11 & $\begin{array}{c}\text { amphore } \\
\text { massaliète } \\
\text { impériale }\end{array}$ & $\begin{array}{c}\text { bord } \\
\text { d'amphore }\end{array}$ & 1 & 20.1 \\
\hline
\end{tabular}

Figure 9 : Inventaire typologique des céramiques (phase I). Figure 9: Pottery typological inventory.

kaolinitique type Goudineau F1 est à situer dans le dernier quart du I ${ }^{\text {er }}$ siècle av. J.-C., celle des cruches et des coupes en pâte claire du bassin de l'Argens est peut être un peu plus ancienne (Fig. 10 [5]). Enfin, un bord de plat en céramique modelée micacée de la région de Fréjus, appartenant à la forme 5 de Lucien Rivet (Rivet 1982, 249-250) remonterait au plus tôt aux années 20/30 apr. J.-C. (Fig. 10 [4]).

\section{Les amphores}

L'amphore italique de type Dressel 2/4 (Fig. 10 [7]) est fréquente sur la côte méditerranéenne gauloise depuis le dernier tiers du I ${ }^{\text {er }}$ siècle av. J.-C., jusque dans la première moitié du $\mathrm{I}^{\mathrm{er}}$ siècle apr. J.-C. Les Dressel 7/11 de Marseille (Fig. 20 [1]) et les amphores à huile de Bétique (Dressel 20) apparaissent toutes deux vers les années 30/20 avant notre ère. Les Dressel 2/4 de Tarraconaise sont produites peu après. L'amphore Dressel 2/4 d'origine gauloise (Fig. 10 [6]), représentée par un bord et une anse, est l'amphore la plus tardive du lot. L'analyse à l'œil nu de la pâte la rapproche des productions fréjussiennes. Les premières traces de production de ces amphores à Fréjus remonteraient au dernier tiers du $\mathrm{I}^{\text {er }}$ siècle av. J.-C., dans l'atelier de Saint-Lambert III (Gébara et Béraud 1996, 319). 

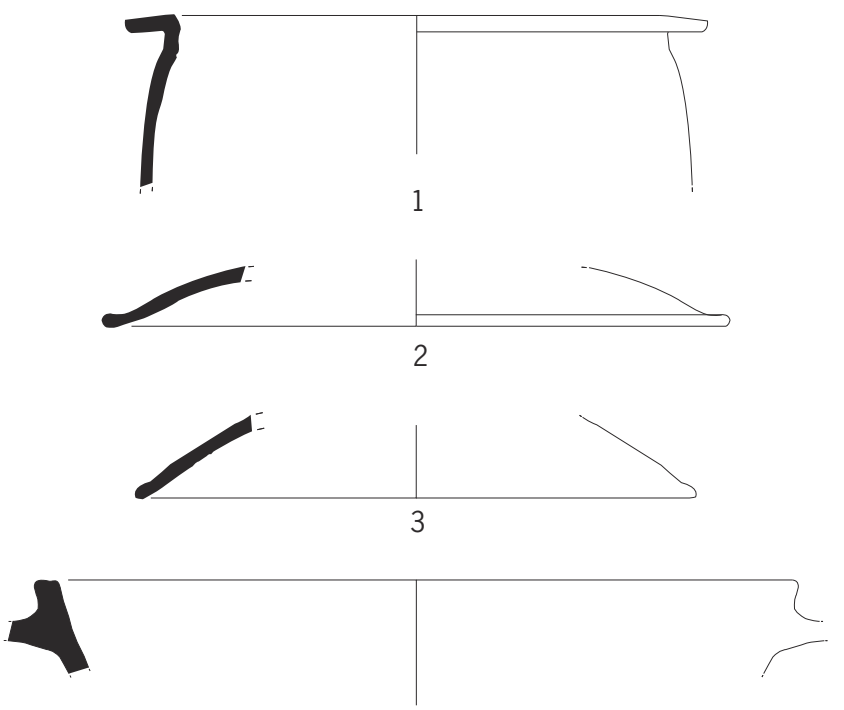

4

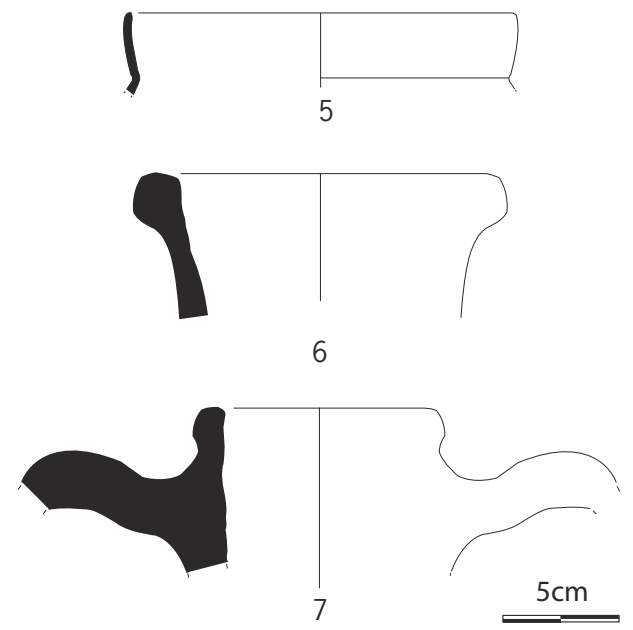

Figure 10 : Planche céramiques provenant de l'US2090 (Phase II) (P. Excoffon).

Figure 10: Pottery coming from the US2090.

\subsubsection{Datation par la céramique et le radiocarbone}

L'ensemble de ce mobilier permet de situer le début de la fréquentation de la côte rocheuse au plus tôt aux alentours des années 30/20 av. J.-C. Concernant la fin de cette phase, les éléments de datation sont moins évidents. Le bord de plat Rivet 5a apparait dans les années 20/30 apr. J.-C., il s'agit de l'élément le plus récent. L'horizon 20/30 pourrait donc correspondre à la fin de cette phase. D'autres arguments a silentio vont dans ce sens, notamment l'absence de fragment de sigillée sud-gauloise, pourtant courante à Fréjus à partir des années 30/40 apr. J.-C.
La datation par radiocarbone de deux vermets encore fixés sur le rocher ${ }^{6}$ et dont la mort des individus composant ce peuplement résulte de l'enfouissement du secteur (échantillons 2 et 10) donne un âge calibré respectivement de 300 B.C. -10 A.D. et de 160 B.C. -80 A.D. avec un intervalle de confiance de $95,4 \%$ (deux sigma) ${ }^{7}$. Cette datation, certes large, n'est pas en opposition avec celle proposée à partir du mobilier céramique, et se joint aux autres éléments permettant d'affirmer l'existence et la position d'un niveau marin antique et ainsi de confirmer l'existence d'une côte rocheuse battue par la mer au pied de la Butte Saint-Antoine lors de la création de la colonie.

\subsubsection{Détermination du niveau marin}

Accrochée au rocher, la faune marine en place (Vermetus Triceter) (US2080) a permis de mesurer le niveau marin antique. En effet, ce type de vermet vit au sommet de la zone infralittorale et marque donc la base de la zone médiolittorale, ce qui correspond au zéro marin biologique (Peres et Picard 1964). L'arrêt de peuplement est de toute évidence provoqué par l'enfouissement du rocher sous les sables littoraux, fossilisant ainsi l'enregistrement biologique du niveau marin. La limite supérieure du peuplement permet donc de mesurer de façon remarquablement précise le niveau marin durant cette période de l'Antiquité romaine, grâce au peuplement biologique associé aux conditions d'enfouissement. Cette limite se situe dans une fourchette entre $-0,210 \mathrm{~m}$ (prél. n 2) et -0,392 m (prél. n 1) NGF (Fig. 11).

La comparaison du niveau marin antique reconnu sur cette opération avec les données régionales de précision (Fig. 12) ne montre pas de différence significative. Le relèvement du niveau marin depuis cette période est tout au plus de $40 \mathrm{~cm}$, ce qui souligne l'importance des apports sédimentaires dans la métamorphose des paysages littoraux durant la fin de l'Holocène. Ceci tend aussi à prouver que malgré la présence de failles, le secteur de Fréjus n’a pas été affecté par des mouvements tectoniques significatifs depuis l'époque romaine.

En arrière de cette portion de côte rocheuse au pied de la butte Saint Antoine, un cordon littoral existe, puisqu'il est mis en évidence pour la période directement postérieure au I ${ }^{\text {er }}$ siècle av. J.-C. Bien qu'il ne soit pas encore localisé avec exactitude, nous pouvons le situer entre l'emprise du chantier du théâtre d'agglomération et le pont des Esclapes,

6. Datations réalisées par Tomasz Goslar du Poznań Radiocarbon Laboratory - Pologne.

7. Prélév 2: 68.2\% probability, 190BC/50BC; $95.4 \%$ probability, 300BC/10AD, ref : Poz-14371. Prélév 10 : 68.2\% probability, 100BC/40AD; $95.4 \%$ probability, 160BC/80AD, ref : Poz-14372 
FRÉJUS

Théâtre d'agglomération

$\mathrm{E}-\mathrm{E}^{\prime}(\mathrm{T} 2)$

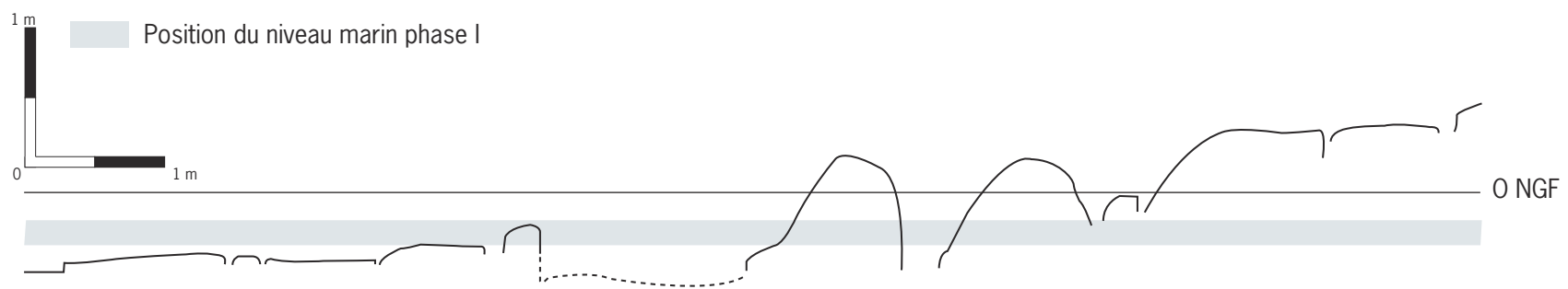

Figure 11 : Coupe du rocher et localisation altimétrie du niveau marin (P.Excoffon/J. Pâques).

Figure 11: Rock section and marine level position.

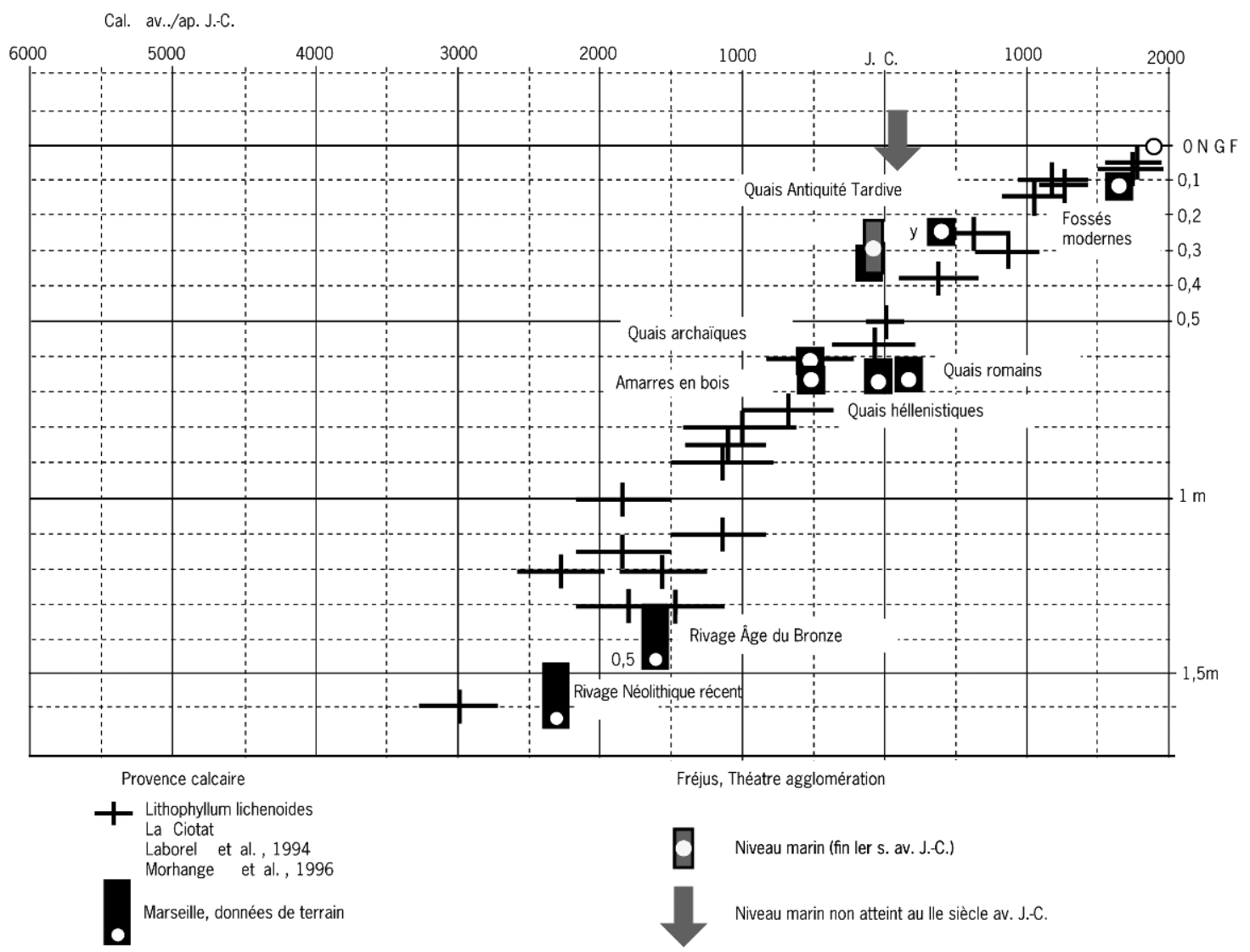

Figure 12: Le niveau marin antique à Fréjus et comparaisons régionales (B. Devillers).

Figure 12: The ancient marine level in Fréjus and local comparisons.

ce secteur étant émergé durant l'antiquité (voir Allinne et Devillers dans ce volume). L'extension de la lagune mise en évidence aux Esclapes durant cette période n'est pas encore connue, mais il est possible qu'elle puisse s'appuyer sur le flanc occidental du promontoire sur lequel la ville de Fréjus est installée.
Une illustration actuelle analogue peut être invoquée avec la côte rocheuse entre Santa Lucia et la plage de l'Homme Mort à Saint-Raphaël (Fig. 13). La morphologie de la côte, les roches affleurantes, les marques d'érosions et même la pollution anthropique évoquent de façon troublante les milieux littoraux antiques découverts ici. 


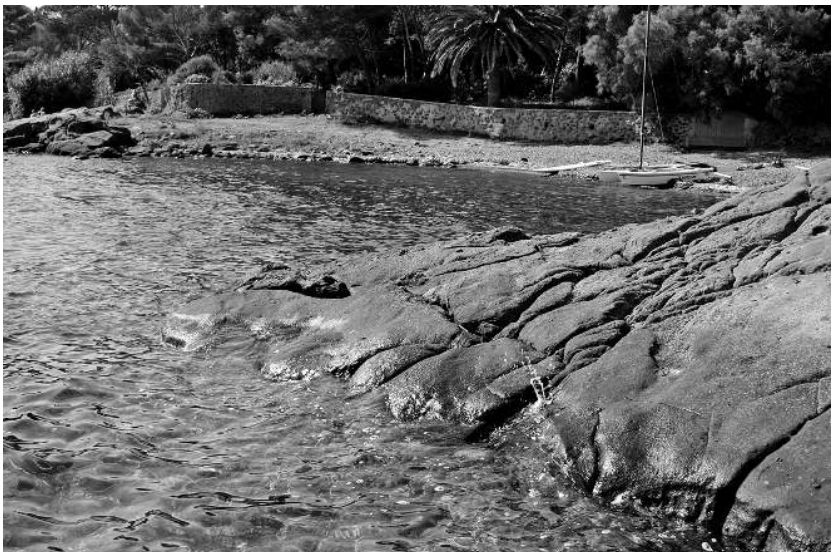

Figure 13 : Côte actuelle, plage de l'Homme Mort, (Saint-Raphaël, Var) (J. Pâques).

Figure 13: Current coast, beach of "l'Homme Mort" (Saint-Raphaël, Var).

Compte-tenu de la fourchette large fournie par le mobilier et les datations radiocarbones, la fréquentation de cette côte peut être placée à partir du dernier tiers du $\mathrm{I}^{\mathrm{er}}$ siècle av. J.-C. Cette nature du littoral et sa position sont un point d'appui nouveau concernant la connaissance du rivage antique à Fréjus. En effet, les publications antérieures situent ce rivage romain plus en aval (Gébara et Chouquer 1996, Cohen 1991 et 1997) ou plus en amont (Dubar 2004) (Fig. 15). Les milieux lagunaires souvent représentés en avant de l'enceinte du port deviennent, grâce à ces nouvelles découvertes, obsolètes.

\subsubsection{Apports de la carpologie à la restitution du paysage végétal local aux alentours du changement d'ère}

Les restes carpologiques provenant des rochers côtiers (US 2090) apportent des informations partielles sur le milieu végétal littoral de Fréjus à la fin du I $\mathrm{I}^{\mathrm{er}}$ siècle av. et au début du $\mathrm{I}^{\text {er }}$ siècle apr. J.-C. (Fig. 14). Il est permis de considérer que ces témoins ont avant tout une valeur locale, même si un apport par les eaux à plus longue distance ne peut pas être exclu, de même qu'un apport par l'homme avec les déchets piégés entre les rochers aux côtés des semences (tessons de céramique, de verre, matériaux de construction, vestiges métalliques, osseux...).

Le milieu aquatique est mis en évidence par deux taxons, Chara sp. et Potamogeton sp. Ceux-ci ne sont toutefois pas typiques des prairies sous-marines mais plutôt des eaux permanentes continentales.

Les bords des eaux, humides, sont évoqués par plusieurs taxons, dont l'aulne (Alnus glutinosa), caractéristique des forêts alluviales qui se développent sur ces sols périodique-

\begin{tabular}{|c|c|c|c|c|c|}
\hline \multicolumn{2}{|l|}{ Tranchée } & 2 & 2 & 2 & \\
\hline \multirow{2}{*}{\multicolumn{2}{|c|}{$\begin{array}{l}\text { Fait } \\
\text { US }\end{array}$}} & & & 2100 & Total \\
\hline & & 2090 & 2090 & 2120 & \\
\hline \multicolumn{2}{|l|}{ Contexte } & sédim & sédim & fosse & \\
\hline \multicolumn{2}{|l|}{ Datation } & fin $\mathrm{I} B C / \mathrm{I} A D$ & fin $\mathrm{I} B C / \mathrm{I} A D$ & IIe $\mathrm{AD}$ & \\
\hline \multicolumn{2}{|l|}{ Volume tamisé } & 3 & 1,5 & 3 & 7,5 \\
\hline \multicolumn{6}{|l|}{ TAXONS ATTESTES } \\
\hline Vicia type & sem. & 5 & - & - & 5 \\
\hline \multicolumn{6}{|l|}{$\begin{array}{l}\text { Restes non carbonisés } \\
\text { Arbres fruitiers }\end{array}$} \\
\hline \multirow[t]{2}{*}{ Ficus carica } & sem. & 26 & 25 & - & 51 \\
\hline & fg. & 10 & 20 & - & 30 \\
\hline Olea europaea & sem. & 1 & - & - & 1 \\
\hline \multirow{2}{*}{\multicolumn{6}{|c|}{ Messicoles }} \\
\hline & & & & & \\
\hline Fallopia convolvulus & sem. & 1 & - & - & 1 \\
\hline \multirow[t]{2}{*}{ Reseda phyteuma } & sem. & - & - & 45 & 45 \\
\hline & fg. & - & 5 & 27 & 32 \\
\hline \multicolumn{6}{|c|}{ Adventices printemps/cultures sarclées } \\
\hline Anagallis arvensis & sem. & 5 & 10 & - & 15 \\
\hline Chenopodium album & sem. & 5 & 5 & - & 10 \\
\hline Euphorbia helioscopia & fg. & - & 5 & - & 5 \\
\hline \multirow[t]{2}{*}{ Fumaria officinalis } & sem. & - & - & 9 & 9 \\
\hline & fg. & - & 5 & - & 5 \\
\hline \multirow[t]{2}{*}{ Heliotropium europaeum } & sem. & - & - & 54 & 54 \\
\hline & fg. & - & 5 & 18 & 23 \\
\hline Portulaca oleracea & sem. & 15 & 10 & - & 25 \\
\hline \multicolumn{6}{|c|}{ Friches, lieux rudéraux, décombres } \\
\hline Polygonum aviculare & fg. & 5 & - & - & 5 \\
\hline \multicolumn{6}{|l|}{ Pelouses, lieux secs } \\
\hline Hypericum perforatum & sem. & - & 10 & - & 10 \\
\hline Petrorhagia prolifera & sem. & - & 5 & - & 5 \\
\hline Potentilla sp. & sem. & - & 5 & - & 5 \\
\hline \multirow[t]{2}{*}{ Saponaria sp. } & sem. & - & 10 & - & 10 \\
\hline & fg. & 5 & 5 & - & 10 \\
\hline Silene cf. gallica & sem. & - & 20 & - & 20 \\
\hline \multicolumn{6}{|l|}{ Bois, lisières } \\
\hline Carpinus betulus & sem. & - & 1 & - & 1 \\
\hline Corylus avellana & fg. & - & 3 & - & 3 \\
\hline Pinus pinea & fg. & 1 & - & - & 1 \\
\hline & écaille & 1 & 3 & - & 4 \\
\hline Rosa/Rubus & épine & - & 1 & - & 1 \\
\hline Rubus fruticosus & sem. & 5 & 6 & - & 11 \\
\hline Rubus sp. & fg. & 26 & 55 & - & 81 \\
\hline Sambucus nigra & sem. & - & 5 & - & 5 \\
\hline Sambucus sp. & fg. & 10 & - & - & 10 \\
\hline Forêt riveraine & & & & & \\
\hline Alnus glutinosa & fg. strobile & - & 2 & - & 2 \\
\hline Euphorbia amygdaloides & sem. & - & 1 & - & 1 \\
\hline Bords des eaux & & & & & \\
\hline Euphorbia cf. exigua & sem. & 5 & 10 & - & 15 \\
\hline Polygonum lapathifolium & fg. & 5 & - & - & 5 \\
\hline Plantes aquatiques & & & & & \\
\hline Chara sp. & oogone & - & 5 & - & 5 \\
\hline Potamogeton sp. & sem. & - & 6 & - & 6 \\
\hline Divers & & & & & \\
\hline Viola sp. & fg. & 5 & 5 & - & 10 \\
\hline Total & & 136 & 251 & 153 & 540 \\
\hline
\end{tabular}

Figure 14 : Fréjus, Théâtre d'Agglomération, résultats carpologiques pour la période antique.

Figure 14: Fréjus, Théâtre d'Agglomération, carpologic results for the ancient period.

ment inondés. Le charme (Carpinus betulus), le noisetier (Corylus avellana) et le sureau noir (Sambucus nigra) sont des ligneux mésophiles qui, en région méditerranéenne, affectionnent également la fraîcheur des forêts riveraines mais, à la différence de l'aulne, sur des sols bien drainés.

Pour sa part, le pin pignon (Pinus pinea), se trouvait probablement sur les sables littoraux. Il est peut être spontané dans la région de Fréjus (Quézel et Médail 2003) mais il a pu également être planté à l'époque romaine pour ses propriétés alimentaires et symboliques (André 1981). L'existence locale de plantations d'arbres fruitiers est par ailleurs fortement suggérée par l'attestation du figuier (Ficus carica), de l'olivier (Olea europaea) et de la vigne (Vitis vinifera). Bien sûr, les 


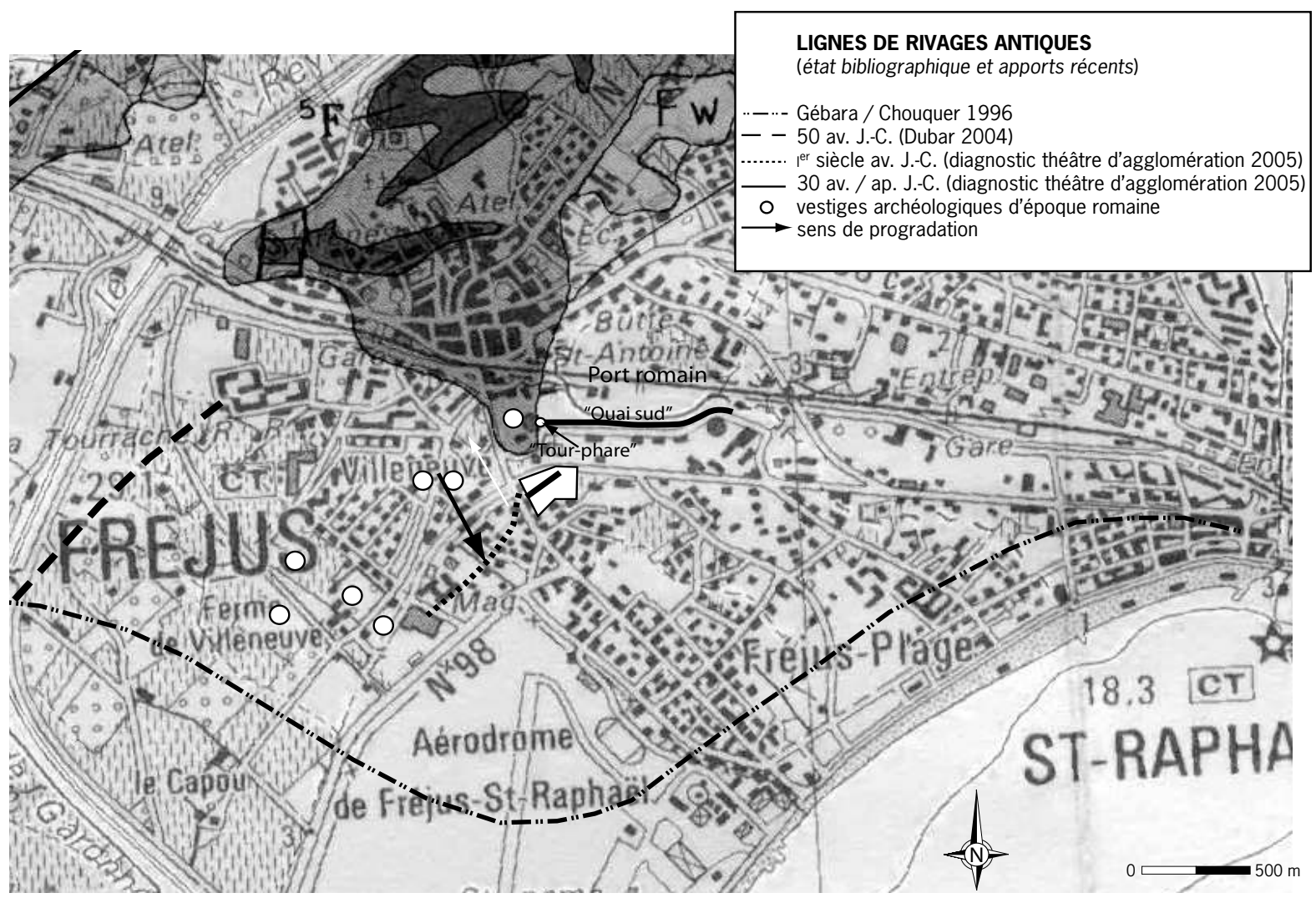

Figure 15 : Carte restituant les différentes hypothèses et le trait de côte induit par le diagnostic du théâtre d'agglomération (P. Excoffon/B. Devillers/ K.-Y. Cotto/J. Pâques).

Figure 15: Map the various assumptions and the coast feature induced by the "Théâtre d'Agglomération" survey.

critères morphologiques directement accessibles à l'observation n'autorisent pas, ou difficilement, la discrimination entre les formes sauvages et cultivées de ces essences pérennes. Pour autant que l'on puisse en juger, les fragments de pépins de raisin évoquent néanmoins franchement la vigne cultivée. Tous les indices archéobotaniques, archéologiques et historiques convergent pour désigner le littoral provençal comme zone d'oléiculture précoce dans le midi de la France, peut être dès le III ${ }^{\mathrm{er}}$ siècle av. J.-C. (Leveau et al. 1991).

L'action de l'homme est également enregistrée dans le spectre carpologique par la fréquence et la variété des plantes adventices et rudérales (neuf taxons au total, Fig. 14), qui témoignent des perturbations du sol et de son enrichissement en matières azotées. Enfin, un petit cortège de cinq plantes herbacées évoque la maigre végétation des espaces secs et ouverts de type pelouse.

\subsection{Phase II La progradation du rivage}

\subsubsection{Description}

Cette deuxième phase correspond à l'avancée du cordon littoral par un ensablement progressif du rocher qui se poursuit au-delà de l'extrémité méridionale des fouilles. Ce processus se traduit par la présence d'une épaisse couche de sable pur, au litage marqué, très régulier et parallèle (pas d'entrecroisement) qui s'épaissit légèrement vers l'aval (Ouest). La granulométrie des sables est variable selon les différents lits, le spectre granulométrique va des sables moyens aux granules. Ce type de lithofaciès associé aux biofaciès (vermets sur le rocher, faune marine remaniée dans le sédiment) indique clairement que le milieu de sédimentation est la zone de swash (zone de déferlement des vagues) de la face externe d'un cordon littoral (Short 2001). Cette unité comprend aussi de nombreux tessons roulés (US2050 et 2060). L'apport graduel de sable en lits successifs, montrant un pendage significatif vers le sud/est, apparait nettement dans la tranchée 2 (Fig. 16) (GEO2040). Les carottages manuels complémentaires ont permis d'estimer une épais- 


\section{FRÉJUS}

Théâtre d'agglomération

Tranchée 2

est / ouest

restitution de la coupe d'ensemble de la tranchée 2, à partir des coupes de terrain BB' et CC'

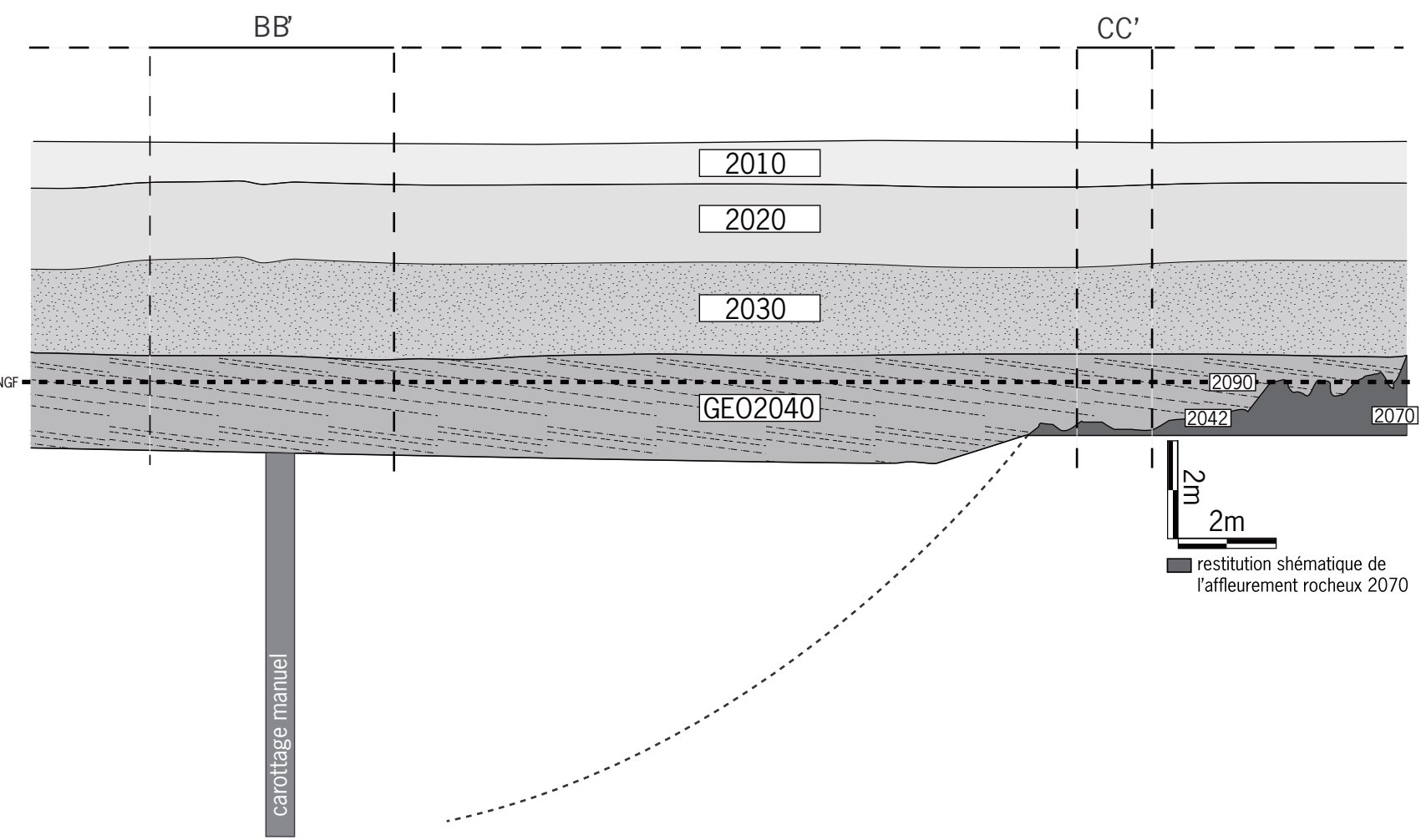

Figure 16 : Coupe est/ouest dans la tranchée 2 (S. Bonnet/B. Devillers/P. Excoffon/J. Pâques).

Figure 16: Section est/west in trench 2.

seur maximale du corps sableux progradant à $5,50 \mathrm{~m}$ vers la partie ouest de la tranchée où le rocher observe une pente importante. Malheureusement le fluage des sables au sein du carottier a limité les possibilités de sondage.

\subsubsection{Sens de progradation et nature de la morphogenèse}

Le pendage des lits sableux, observé sur deux tranchées perpendiculaires, permet de mesurer le sens de progradation de ce littoral deltaïque. Le pendage est fort dans la tranchée T1 alors que dans la tranchée $\mathrm{T} 2$ le pendage est faible vers l'est. La progradation du cordon littoral s'effectue donc dans le sens NNO-SSE. Cette dernière observation tend à montrer que la nature de la dynamique du cordon littoral sur le site de l'opération archéologique est d'origine fluviatile (apports sédimentaires de l'Argens). L'hypothèse d'une flèche sableuse formée par la dérive littorale (hypothétiquement de sens NEE-SOO) ayant pour accroche le promontoire rocheux de la butte SaintAntoine doit donc être ici écartée.
Le budget sédimentaire est donc largement positif durant cette période. Autrement dit, le point d'équilibre déterminé par la position du trait de côte (importance croissante des agents météo marins au débouché de la vallée) et par l'apport de sédiments dépendant des fluctuations climatiques et de l'état des sols, n'est pas encore atteint. Durant cette phase, les indices de l'occupation humaine sont composés de quelques artefacts ${ }^{8}$, aucune perturbation d'origine anthropique n'a été perçue dans les sondages. Il semble que le secteur corresponde à une plage de sable sans occupation manifeste. Toutefois, c'est durant cette phase que s'établit en arrière de la plage la maison romaine découverte en 1994, à moins de $100 \mathrm{~m}$ au nord du secteur sondé.

Cette avancée du trait de côte se réalise sous l'influence des apports sédimentaires de l'Argens, comme le montrent les pendages et la direction générale du sens de prograda-

8. Sur le rocher, le recouvrement sableux a livré quelques tessons (51 frgts), des fragments d'imbrices et de tegulae (4) et des clous de section carrée (9). 
tion. Ce rivage peut être mis directement en relation avec l'existence de lagunes reconnues plus en amont (Allinne \& Devillers, dans ce volume). L'extension spatiale de ce type de milieu n'est pas encore connue, les analyses en cours (étude de l'ostracofaune et transect électrique) permettront éventuellement d'en signaler la présence en amont du site (secteur ouest de la ville) durant l'Antiquité romaine.

\subsubsection{Datation}

Les éléments de datation de cette phase sont fournis principalement par les US 2050 et 2060 (Fig. 17). Le bord d'assiette Conspectus 4.3 en sigillée italique se place entre 15 av. J.-C. et 15 apr. J.-C., tandis que la coupe Dragendorff $27 \mathrm{~b}$ en sigillée sud-gauloise est produite entre 40 et 80 apr. J.-C. Enfin, l'amphore Gauloise 4 de Fréjus n'existe pas avant le milieu du I $\mathrm{I}^{\text {er }}$ siècle apr. J.-C. Dans ces sables a également été découvert un bord d'amphore Dressel IB, à l'évidence résiduel, mais qui constitue le tesson le plus ancien découvert lors de la fouille et qui pourrait remonter aux années 50/40 av. J.-C.. Le début de cette deuxième phase est très complexe à définir compte tenu de sa nature. Nous proposons toutefois de le situer vers les années 15 apr. J.-C., l'élément

\begin{tabular}{|c|c|c|c|}
\hline Zone 2 & US 2050 & & \\
\hline Typologie & Catégorie & forme & NMI \\
\hline KAOL F1 & $\begin{array}{l}\text { céramique } \\
\text { kaolinitique }\end{array}$ & $\begin{array}{l}\text { anse de } \\
\text { cruche }\end{array}$ & 1 \\
\hline $\begin{array}{c}\text { CNT-VAR } \\
\text { Bérato } 651\end{array}$ & $\begin{array}{l}\text { modelée } \\
\text { varoise }\end{array}$ & bord de jatte & 1 \\
\hline $\begin{array}{c}\text { SIG-IT CONSP } \\
4.3\end{array}$ & \multirow{2}{*}{$\begin{array}{c}\text { céramique } \\
\text { sigillée italique }\end{array}$} & bord d'assiette & 1 \\
\hline SIG-IT R4.2 & & bord de calice & 1 \\
\hline SIG-SG Dr27b & $\begin{array}{l}\text { céramique } \\
\text { sigillée sud- } \\
\text { gauloise }\end{array}$ & $\begin{array}{l}\text { bord de } \\
\text { coupelle }\end{array}$ & 1 \\
\hline A-GAUL 4 & $\begin{array}{l}\text { amphore } \\
\text { gauloise }\end{array}$ & $\begin{array}{c}\text { bord } \\
\text { d'amphore }\end{array}$ & 1 \\
\hline Zone 2 & US 2060 & & \\
\hline Typologie & Catégorie & forme & NMI \\
\hline COM-GRE 1d & $\begin{array}{c}\text { céramique } \\
\text { commune } \\
\text { grecque }\end{array}$ & bord de chytra & 1 \\
\hline $\begin{array}{c}\text { CNT-VAR } \\
\text { Bérato } 154\end{array}$ & $\begin{array}{l}\text { modelée } \\
\text { varoise }\end{array}$ & bord d'urne & 1 \\
\hline $\begin{array}{c}\text { CL-IMPRO } \\
\text { Pasq B3 }\end{array}$ & \multirow{2}{*}{$\begin{array}{l}\text { Pâte claire } \\
\text { provençale }\end{array}$} & bord de cruche & 1 \\
\hline CL-IMPRO & & bord de vase & 1 \\
\hline A-BET Dr7/11 & $\begin{array}{c}\text { amphore de } \\
\text { Bétique }\end{array}$ & $\begin{array}{c}\text { bord } \\
\text { d'amphore }\end{array}$ & 1 \\
\hline A-ITA Dr1B & $\begin{array}{c}\text { amphore } \\
\text { italique }\end{array}$ & $\begin{array}{c}\text { bord } \\
\text { d'amphore }\end{array}$ & 1 \\
\hline A-M-I 7a & $\begin{array}{c}\text { amphore } \\
\text { massaliète } \\
\text { impériale }\end{array}$ & $\begin{array}{c}\text { bord } \\
\text { d'amphore }\end{array}$ & 1 \\
\hline
\end{tabular}

Figure 17 : Inventaire typologique des céramiques (phase II). Figure 17: Pottery typological inventory (phase II). de datation le plus tardif témoignant de la progradation du rivage est attribué à la seconde moitié du $\mathrm{I}^{\mathrm{er}}$ siècle.

\subsubsection{Rapidité de la progradation durant l'époque romaine et impact anthropique sur le détritisme}

La vitesse de progradation ne peut être précisément évaluée durant l'époque romaine. Il est tentant d'évoquer que celle-ci ait été favorisée par l'implantation de colons romains entreprenant une mise en valeur agricole du territoire de Forum Julii et provoquant une érosion accélérée des sols dans le sens décrit par Neboit-Guilhot (1999), ce phénomène est décrit pour cette période dans le bassin Valdenais par exemple (Berger 1995). Toutefois des points de comparaison ne peuvent encore être établis pour la vallée. En effet, ce type de comparaison nécessite une chronologie très précise de l'activité détritique d'une part, et de la progression du trait de côte d'autre part. De plus, ces données doivent être obligatoirement comparées à la morphologie de la vallée ante-holocène qui détermine l'espace d'accommodation de la ria (volume à remblayer pour permettre la progradation). Ces données sont malheureusement encore lacunaires. Enfin, certains auteurs (Bravard et al. 1990, Devillers et Provansal 2002, Bruneton et al. 2003 par exemple...) signalent la faiblesse de l'activité détritique durant la période romaine et l'expliquent par la bonne gestion des eaux de ruissellement couplée à une phase climatique généralement peu agressive envers les sols. Si l'opération préventive du théâtre d'agglomération apporte des informations sur les rapports entre la gestion des terroirs, le détritisme et l'évolution des milieux littoraux, elle ne permet nullement d'apporter une conclusion sur ce sujet.

\subsection{Phase III : la phase aggradante}

\subsubsection{Description}

Cette phase est marquée dans la stratigraphie par une couche sédimentaire d'une épaisseur variant entre 0,57 et un mètre selon les tranchées. Elle se compose de sables roux moyens et fins, homogènes, assez bien triés, sans structure sédimentaire ou pédologique et très légèrement enrichis en limons. Ce sable a été repéré dans toutes les tranchées, mais il est clairement apparu dans les tranchées T1, T2 et T5, respectivement US $1030,2030^{\circ}$ et $5040^{10}$. La granulométrie des sables montre la disparition de l'asymétrie négative, en faveur d'une répartition gaussienne, voire d'une légère asymétrie positive (0,1 Skewness), témoignant de la disparition des processus de lessivage et d'un milieu émergé (Fig. 18).

9. niv. sup. 1,24 $\mathrm{m}$ NGF.

10. niv. sup. 0,72 $\mathrm{m}$ NGF. 


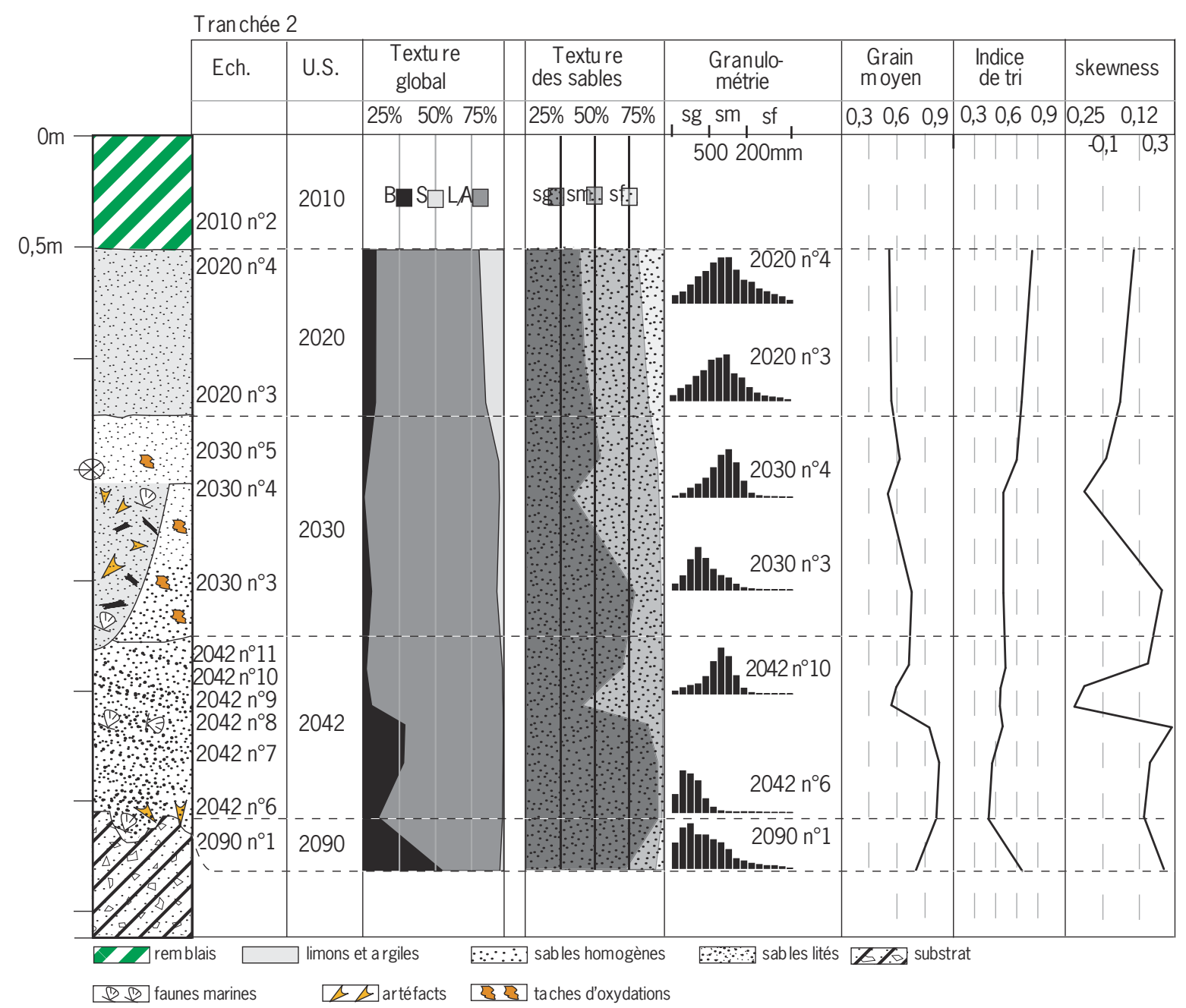

Figure 18 : Stratigraphie et analyses sédimentologiques de la coupe T2 (S. Bonnet/B. Devillers).

Figure 18: Stratigraphy and sedimentological analysis of T2 section.

L'interprétation paléoenvironnementale de cette unité doit donc être rapprochée d'un haut de plage, soumis au ruissellement provenant de la butte Saint-Antoine (apports colluviaux de sédiments fins à partir du substrat permien) et enrichi en apports sableux par l'activité éolienne.

Ces sédiments témoignent de l'éloignement relatif du trait de côte. Comme pour les niveaux de sable lité, la surface du sable a un pendage vers le sud. D'un point de vue stratigraphique, il faut toutefois noter que l'évolution taphonomique de cette phase s'effectue ici de façon aggradante par rapport aux phases précédentes marquées par les processus de progradation. Il en résulte une légère ambiguïté que les tranchées effectuées n'ont pas permis de résoudre, les dernières phases progradantes (au sud/est du site) pouvant correspondre chronologiquement aux premiers dépôts aggradants de la phase III dans le secteur nord du chantier. En d'autres termes, l'aggradation de l'arrière plage par colluvionnement de la Butte Saint-Antoine est synchrone de l'avancée du trait de côte sous l'influence des apports sédimentaires de l'Argens.

C'est à partir de ce niveau que les premiers aménagements d'origine anthropique ont été repérés. Il s'agit essentiellement d'une fosse circulaire (FS2100) creusée dans le niveau de sable roux (US 2030). De forme tronconique, son diamètre maximal mesure $100 \mathrm{~cm}$, pour une profondeur de $75 \mathrm{~cm}$. La surface de creusement se situe à $0,95 \mathrm{~m}$ NGF. Le comblement de la fosse est composé de deux niveaux distincts (US 2120 et 2130). La couche supérieure de remblai, 2120, est faite d'un limon sableux mélangé à de la chaux. Des tessons de céramiques ainsi que deux clous ont été retrouvés. La couche inférieure (2130) est composée de limons sableux 
très compacts mélangés à des nodules de chaux. De la céramique, quelques coquillages (dont des murex) et des os ${ }^{11}$ sont présents.

\subsubsection{Datation}

Les éléments de datation pour cette phase sont peu nombreux, le niveau de sable roux a livré quelques tessons, notamment l'US 2030 dans la tranchée 2 (Fig. 19). Malheureusement ceux-ci ne permettent pas une datation précise du contexte. Le faitout 651 (Bérato 1993, 330) est diffusé entre le Ier et le Iv $v^{e}$ siècle apr. J.-C. et les cruches en pâte kaolinitique type $\mathrm{F} 1$ sont produites au moins jusqu'à la fin du $\mathrm{II}^{\mathrm{e}}$ siècle. Ce niveau a également livré une anse complète d'amphore à huile de Bétique Dressel 20 comportant dans sa partie haute une estampille. Il s'agit d'un cartouche rectangulaire $(5,8 \times 2,2 \mathrm{~cm})$ en relief très érodé. Les deux premières lettres sont nettement visibles, un $S$ suivi d'un $A$,

\begin{tabular}{|c|c|c|c|c|}
\hline Zone 2 & US 2030 & & & \\
\hline Typologie & Catégorie & forme & NMI & Figure \\
\hline KAOL F1 & $\begin{array}{l}\text { céramique } \\
\text { kaolinitique }\end{array}$ & bord de cruche & 1 & \\
\hline $\begin{array}{c}\text { CNT-VAR } \\
\text { Bérato } 651\end{array}$ & $\begin{array}{l}\text { Modelée } \\
\text { varoise }\end{array}$ & bord de jatte & 1 & \\
\hline A-BET Dr20 & $\begin{array}{c}\text { amphore de } \\
\text { Bétique }\end{array}$ & $\begin{array}{c}\text { anse } \\
\text { d'estampille }\end{array}$ & 1 & \\
\hline Zone 2 & US 2110 & & & \\
\hline Typologie & Catégorie & forme & NMI & Figure \\
\hline A-BET Dr20B & $\begin{array}{l}\text { amphore de } \\
\text { Bétique }\end{array}$ & $\begin{array}{c}\text { bord } \\
\text { d'amphore }\end{array}$ & 1 & 20. 2 \\
\hline Zone 2 & US 2130 & & & \\
\hline Typologie & Catégorie & forme & NMI & Figure \\
\hline COM-IT 7c & \multirow{3}{*}{$\begin{array}{c}\text { céramique } \\
\text { commune } \\
\text { italique }\end{array}$} & \multirow{2}{*}{$\begin{array}{c}\text { bord de } \\
\text { couvercle }\end{array}$} & 1 & \\
\hline COM-IT 7e & & & 1 & \\
\hline COM-IT n. c & & bord d'urne & 1 & 20.3 \\
\hline CNT-VAR Riv3 & $\begin{array}{c}\text { Modelée } \\
\text { varoise }\end{array}$ & bord de plat & 1 & \\
\hline R-POMP 15 & $\begin{array}{c}\text { céramique à } \\
\text { engobe rouge } \\
\text { interne } \\
\text { pompéien }\end{array}$ & fond de plat & 1 & \\
\hline SABL-OR & $\begin{array}{c}\text { céramique } \\
\text { sableuse } \\
\text { oxydante }\end{array}$ & bord de vase & 1 & \\
\hline SIG-IT & $\begin{array}{c}\text { céramique } \\
\text { sigillée italique }\end{array}$ & fonds de vase & 2 & \\
\hline SIG-SG Dr27c & \multirow[t]{2}{*}{$\begin{array}{l}\text { céramique } \\
\text { sigillée sud- } \\
\text { gauloise }\end{array}$} & $\begin{array}{l}\text { bord de } \\
\text { coupelle }\end{array}$ & 1 & \\
\hline SIG-SG & & $\begin{array}{c}\text { fond } \\
\text { d'estampille }\end{array}$ & 1 & \\
\hline A-GAUL 11 & \multirow[t]{2}{*}{$\begin{array}{l}\text { amphore } \\
\text { gauloise }\end{array}$} & $\begin{array}{c}\text { fond } \\
\text { d'amphore }\end{array}$ & 1 & \\
\hline A-GAUL 2 & & $\begin{array}{c}\text { bord } \\
\text { d'amphore }\end{array}$ & 1 & 20.4 \\
\hline A-GRE Cret02 & $\begin{array}{c}\text { amphore } \\
\text { grecque }\end{array}$ & $\begin{array}{c}\text { bord } \\
\text { d'amphore }\end{array}$ & 1 & 20.5 \\
\hline
\end{tabular}

Figure 19 : Inventaire typologique des céramiques (phase III). Figure 19: Pottery typological inventory (phase III).

11. Il s'agit d'une moitié proximale de métatarse III de porc, de trois esquilles indéterminées et d'un élément indéterminé de couleur blanche : os ou coquille (Identification Isabelle Rodet-Belarbi). ainsi que les deux dernières, un $\mathrm{E}$ et un $\mathrm{R}$. L'espace illisible devait être utilisé par au moins trois lettres. Par recoupement (type d'amphore, format de l'estampille) la marque la plus proche est SAX.FER se développant en $\operatorname{Sax}($ o) Fer(reo). Dans ce cas le lieu de production serait à situer à Huerta de Belén (Callender 1965 n $^{\circ}$ 1573, 242; Amar et Liou 1989, 200; Blanc-Bijon et al. 1998, n 1234, 236).

Le comblement de la fosse FS2100 (2110 et 2130) (Fig. 20 [2 à 6]) a livré du matériel, dont l'élément le plus récent est un bord de coupelle en sigillée sud-gauloise type Dragendorff 27c, datée entre 80 et 120 apr. J.-C.

La mise en place des sables roux recouvrant les niveaux lités

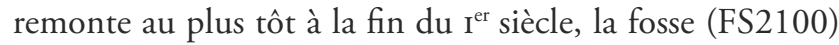
creusée dans ces sables, et recouverte par ces mêmes sables, est comblée après 80 , donc au plus tôt dans le dernier quart $\mathrm{du} \mathrm{I}^{\mathrm{er}}$ siècle. Nous proposons de situer cette phase entre le dernier tiers du I $\mathrm{I}^{\mathrm{er}}$ et le $\mathrm{II}^{\mathrm{e}}$ siècle apr. J.-C., sans plus de précision quant à la fin de cette phase.

\section{Conclusion}

La principale découverte de cette campagne de sondages réside dans la détermination précise de la nature, de la position et de l'évolution du littoral antique, contemporain de l'implantation de la colonie. Au I $\mathrm{e}^{\mathrm{er}}$ siècle av. J.-C., les terrains situés immédiatement au sud de la Butte Saint-Antoine sont constitués d'une bande étroite d'environ $100 \mathrm{~m}$ de large, ponctuée au sud par saillant rocheux. Cette proximité de la mer avec la Butte Saint-Antoine permet de considérer d'un regard nouveau l'aménagement et l'occupation, semble-t-il privilégiée, de ce secteur de la ville. Il s'agit, ne l'oublions pas, du secteur où la présence humaine la plus ancienne, datée du premier Âge du Fer, a été mise en évidence par P.-A. Février et M.-E. Bellet entre 1974 et 1976 (Rivet 2000, 280281). Les indices de site étaient constitués d'un mur posé sur un niveau de gros galets de grès roulés. La provenance de ces gros galets pourrait être expliquée par la proximité de la mer. Selon la même logique, sans doute convient-il de reconsidérer la fonction de la "tour-phare ${ }^{12}$ ", relevée et interprétée ainsi par Charles Texier. Les doutes émis sur sa véritable vocation (Rivet 2000, 287) se justifient essentiellement dans une configuration où le littoral se place à plusieurs centaines de mètres plus au sud. La présence d'un véritable cap rocheux nous incite à reconsidérer la question, car l'évidence d'un phare, d'une balise ou d'un amer à cet endroit périlleux, à proximité immédiate de l'accès au port,

12. Il s'agit de la plus haute tour édifiée au sud-est de la Butte SaintAntoine et dominant le port à l'est. 


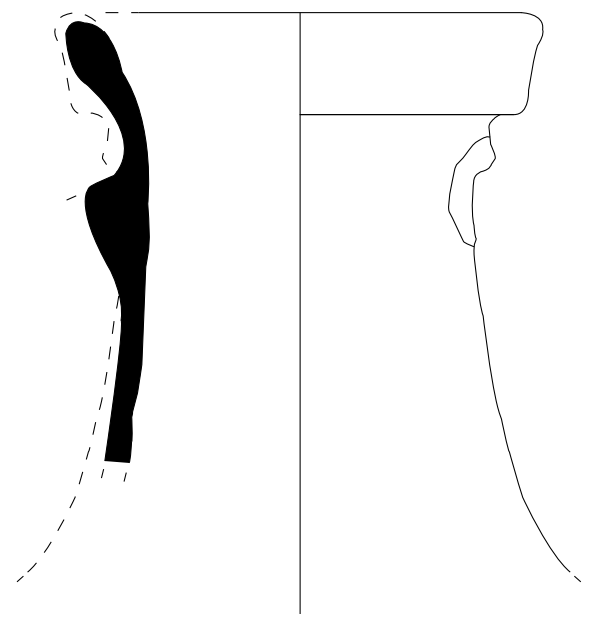

1

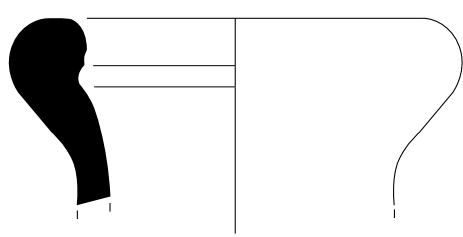

2

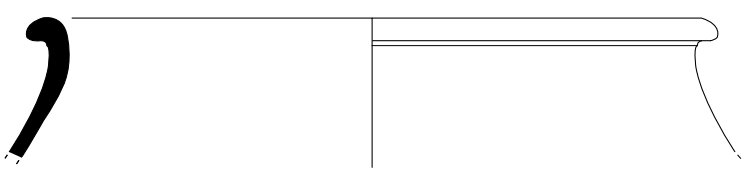

3

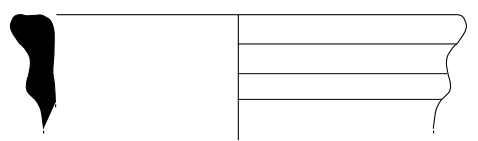

4

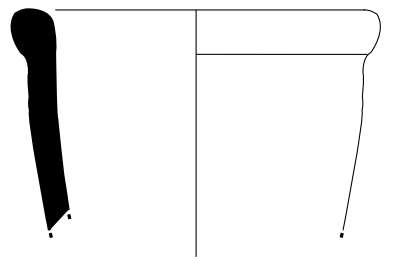

5

s'impose d'elle-même (Fig. 15). La Butte Saint-Antoine constitue le point haut de la côte et représente un endroit idéal pour y installer un tel bâtiment, au point de contact du quai avec la terre ferme.

Cette côte apparaît occupée à partir des années 20 av. J.-C., soit plus de 20 ans après la fondation de Fréjus. La présence de matériaux de construction souillant la côte (tuiles, mortier, enduit, clou...) pourrait indiquer dès cette époque l'existence d'un habitat à proximité immédiate. Enfin, le tracé restituable de la côte dans la partie est remet en cause la longueur supposée du canal oriental d'accès au port antique (février 1963) au moins pour le début du Haut-Empire. De la même façon le rôle du mur de "quai " méridional peut être aujourd'hui envisagé non plus comme un quai, mais comme un mole situé en mer et protégeant le port (Fig. 15). Rien n'est encore établi, mais des études sont désormais à mener en ce sens, surtout en exploitant du mieux possible les opportunités fournies par les aménagements urbains prévus en avant du port antique.

Le ${ }^{\text {er }}$ siècle marque l'avancée rapide du cordon littoral sous l'effet des apports sédimentaires de l'Argens. Les rapports entre l'avancée du trait de côte, les fluctuations des apports sédimentaire (détritisme) et les variations des activités humaines induites par la colonisation romaine ne peuvent être explicités avec les seules données obtenues sur cette opération de diagnostic. Cette thématique ne pourra être abordée qu'à la lumière d'un corpus de donnés suffisamment représentatif de la ria. La construction du port et de son quai sud a aussi pu modifier fortement la répartition initiale des sédiments et ainsi participer à l'accélération du phénomène de progradation. À partir de la fin du $\mathrm{I}^{\text {er }}$ siècle au moins, les terrains gagnés sur la mer ne sont pas bâtis.

Enfin, la fouille permet d'écarter définitivement l'hypothèse d'un chenal d'accès antique au port depuis l'ouest, passant devant le camps de Villeneuve, qu'une trace sombre relevée par photo-interprétation avait permis d'appuyer (Gébara et Chouquer 1996).D’une part sa position se trouve au sud du trait de côte, donc en mer lors de la fondation de la colonie. D'autre part la tranchée T5 prenant cette trace en écharpe, a révélé une zone de limon sableux gris-noir, mis en place dans le courant du $\mathrm{XIX}^{\mathrm{e}}$ siècle, celle-ci semble être la cause de la bande noire, pour laquelle nous n'avons toutefois aucune explication.

Figure 20 : Planche des céramiques provenant de l'US2090 $\left(\mathrm{n}^{\circ} 1\right)$ (Phase II) et US2110 et 2130 (n 2 à 6) (Phase III) (P. Excoffon/H. Garcia).

Figure 20: Pottery coming from the US2090 (1) (Phase II) and US2110 and 2130 (2-6) (Phase III).

ArcheoSciences, revue d'archéométrie, 30, 2006, p. 205-221 


\section{Bibliographie}

Amar, G. et Liou, B., 1989. Les estampilles sur amphores du golfe de Fos (II), SFECAG, Actes du congrès de Lezoux, 4-7 mai 1989, p. 191-207.

André, J., 1981. L'alimentation et la cuisine à Rome, Paris, Les Belles Lettres, $252 \mathrm{p}$.

Bats, M., 1993. Céramique commune italique dans Dicocer. Dictionnaire des Céramiques Antiques (VIT s. av. n. è.-VII s. de n. è.) en Méditerranée nord-occidentale (Provence, Languedoc, Ampurdan), Lattara, 6, p. 357-362.

BérATo, J., 1993. Evolution de la céramique non tournée de la fin de l'Age du fer à la période gallo-romaine dans le département du Var, DAMérid. 16, p. 317-336.

BERGER, J.-F., 1995. Facteurs anthropiques et naturels de l'évolution des paysages romains et protomédiévaux du Bassin valdainais (Drôme), in S. van der Leeuw (dir.), L'Homme et la dégradation de l'environnement, Actes des rencontres, 20-22 octobre 1994, Nice Sophia Antipolis, APDCA, p. 79-114.

Blanc-Bijon, V., Carre, M.-B., Hesnard, A. et Tchernia, A., 1998. Recueil de timbres sur amphores romaines, II, (1989-1990 et compléments 1987-1988), Aix-en-Provence, 1998.

Callender, M.-H., 1965. Roman Amphorae with Index of Stamps, Londres, 1965.

CoHEN, O., 1991. Impacts des aménagements sur la morphologie et l'écologie côtières de la baie de Fréjus, Université de Nice SophiaAntipolis, 1991.

Cohen, O, 1997. La baie de Fréjus : 2000 ans d'évolution du rivage, Mappemonde (1), p. 6-12.

Conspectus, Ettlinger, E., Hedinger, B., Hoffmann, B., Kenrick, P.-M., Pucci, G., Roth-Rubi, K., Schneider,G., Von Schnurbein, S., Wells, C.-M. et Zabehlicky-scheffeNEGGER, S., 1990. Conspectus Formarum tarrae sigillatae italico modo confectae, Materialien zur römisch-germanischen Keramik, 10, Bonn.

Dubar, M., Mai, B.-T. et Pichard, S., 1993. Milieu naturel et anthropisation en Provence cristalline à l'Holocène, in J. L. Fiches, F. Audouze, S Van der Leeuw (dir.), Hommes, espaces et techniques dans la région de Fréjus (Var). Occupation et environnement dans le bassin de l'Argens depuis le Néolithique, ATP Fréjus-Argens, Rapport final 1990-1993, p. 1-24.

Dubar, M., 2004. L'édification de la plaine deltaïque du Bas Argens (Var, France) durant la Protohistoire et l'Antiquité. Application d'un modèle numérique 2D à l'archéologie, Méditerranée, 1(2), p. 47-54.
Février, P.-A, 1963. Le port Antique de Forum Julii, Photo Interprétation, 3, Paris, Bimestriel Mai-Juin 1963 : 43-49.

Gascou, J. et JanON M., 1985. Inscriptions Latines de Narbonnaise (ILN), Fréjus, XLIVe supplément à Gallia, 1985.

Goudineau Ch., 1982. Une fouille récente à la périphérie de Forum Iulii : le chantier des Aiguières, dans Comptes rendus de l'Académie des Inscriptions et Belles-Lettres, 1982, p. 279-292.

Gébara, Ch. et Béraud, I, 1996. Les céramiques communes de Fréjus, production et consommation, in M. Bats (dir.), Les céramiques communes de Campanie et de Narbonnaise (Ir siècle av. J.-C., II siècle apr. J.-C.), La vaisselle de cuisine et de table. Actes des journées d'étude (Naples 1994), Naples, p. 299-325.

Gébara, Ch. et Chouquer, G., 1996. Les parcellaires antiques de la région de Fréjus, in Les trames du paysage, T. 1, Etudes sur les parcellaires, Paris, Errance, coll. "Archéologie Aujourd'hui ", p. 91-103.

Laborel, J. et Morhange, C. et al., 1994. Biological evidence of sea-level rise during the last 4500 years on the rocky coasts of continental southwestern France and Corsica, Marine Geology, 120, p. 203-223.

Leveau, Ph., Heinz, C., Laval, H., Marinval, Ph. et Medus, J., 1991. Les origines de l'oléiculture en Gaule du sud. Données historiques, archéologiques et botaniques, Revue d'Archéométrie, 15, p. 83-94.

Morhange, C. et Laborel, J. et al., 2001. Changes of relative sea level during the past 5000 years in the ancient harbor of Marseilles, Southern France, Palaeogeography, Palaeoclimatology, Palaeoecology, 166, p. 319-329.

Moerman, M., 1994. Fréjus, Pied de la Butte Saint-Antoine,Bilan scientifique du Service Régional de l'Archéologie de PACA (1994), p. 194-195.

Neboit-Guilhot, R., 1999. Autour du concept d'érosion accélérée : l'homme, le temps et la morphogenèse, Géomorphologie, 2, p. 159-172.

QuÉZEL, P. et MÉDAIL, F., 2003. Ecologie et biogéographie des forêts du bassin méditerranéen, Paris, Elsevier, 571 p.

RivET, L., 1982. La céramique culinaire micacée de la région de Fréjus (Var), RANarb, XV, p. 243-262

Rivet, L., Brentchaloff, D., Roucole, S. et Saulnier, S., 2000. Atlas topographique des villes de Gaule Méridionale, 2, Fréjus, $32^{\mathrm{e}}$ suppl. à la RAN, 2000, 509 p.

SHORT, A. D., 2001. Handbook of beach and shoreface morphodynamics, Wiley \& Sons, 2001, 379 p. 